\title{
Détermination de l'accélération de la pesanteur pour la balance du watt du LNE
}

\section{Free fall acceleration determination for the $\mathrm{LNE}$ watt balance}

\author{
Sébastien MERLET ${ }^{1}$, Pierre GILLOT ${ }^{1}$, Tristan FARAH ${ }^{1}$, Quentin BODART ${ }^{1,2}$, Julien LE GOUET ${ }^{1,3}$, \\ Patrick CHEINET ${ }^{1,4}$, Christine GUERLIN ${ }^{1,5}$, Anne LOUCHET-CHAUVET ${ }^{1,4}$, Nicola MALOSSI ${ }^{1,6}$, \\ Alexander KOPAEV ${ }^{7}$, Olivier FRANCIS ${ }^{8}$, Giancarlo D'AGOSTINO ${ }^{9}$, Michel DIAMENT ${ }^{10}$, Gérard GENEVES ${ }^{11}$, \\ André CLAIRON ${ }^{1}$, Arnaud LANDRAGIN ${ }^{1}$ et Franck PEREIRA DOS SANTOS ${ }^{1}$ \\ 1 LNE-SYRTE, Observatoire de Paris, CNRS, UPMC, UMR 8630, 61 avenue de l'Observatoire, 75014 Paris, France, sebastien.merlet@obspm.fr. \\ 2 Adresse actuelle : Dipartimento di Fisica e Astronomia \& LENS, Universita di Firenze, Italie. \\ 3 Adresse actuelle : ONERA, 91761 Palaiseau Cedex, France. \\ 4 Adresse actuelle : LAC, CNRS, UPR 3321, 91405 Orsay, France. \\ 5 Adresse actuelle : LKB, CNRS, UPMC, UMR 8552, 75005 Paris, France. \\ 6 Adresse actuelle : School of Science and technology, Physics Division, University of Camerino, Italie. \\ 7 Sternberg Astronomical Institute, University of Moscow, V-234 Moscow 119992, Russie \\ 8 University of Luxembourg, Luxembourg. \\ 9 INRiM, Italie. \\ 10 Institut de Physique du Globe de Paris, Université Paris Diderot, 75205 Paris, France. \\ 11 LNE-Trappes, 78197 Trappes Cedex, France.
}

\section{Résumé}

Le projet «balance du watt » propose de relier la définition du kilogramme à la constante de Planck. La pesée de la masse impliquée nécessite une détermination de l'accélération de la pesanteur $g$ avec une exactitude meilleure que $10^{-8}$. Les travaux résumés dans cet article visent à réaliser cette détermination à l'aide d'un gravimètre atomique et d'un site gravimétrique dédié.

\section{MOTS CLÉS $\quad$ : GRAVIMÉTRIE, $\quad$ CARTOGRAPHIE} GRAVIMÉTRIQUE, MODÉLISATION GRAVIMÉTRIQUE, INTERFÉROMÉTRIE ATOMIQUE, BALANCE DU WATT.

\footnotetext{
Abstract

The "watt balance" project aims at linking the kilogram definition to the Planck constant. The weighing of the mass involved requires a determination of the acceleration $g$ with an uncertainty better than $10^{-8}$ This work aims at determining $g$ with an atomic gravimeter and a dedicated gravimetric site.

KEY WORDS: GRAVIMETRY, GRAVITY MAPPING, GRAVITY MODEL, ATOMIC INTERFEROMETRY, WATT BALANCE.
}

\section{Introduction}

L'actuelle définition du kilogramme reste la seule des sept unités de base du Système international d'unités (SI) encore basée sur un artefact matériel [1], le prototype international du kilogramme (PIK). Les vérifications périodiques entre le PIK et ses témoins ont révélé une dérive relative moyenne de $5 \times 10^{-8}$ [2] en 100 ans mais la dérive absolue pourrait être encore plus importante. Cette situation est problématique pour le domaine des masses mais également pour les unités qui en dérivent telles que l'ampère, la mole et la candela [3]. Considérant la faiblesse du système actuel, plusieurs recommandations de la Conférence générale des poids et mesures (CGPM) [4-6] incitent les instituts nationaux de métrologie à poursuivre leurs efforts pour affiner les expériences qui relient l'unité de masse à des constantes fondamentales ou atomiques et qui pourraient, dans l'avenir, servir de base à une nouvelle définition du kilogramme. 


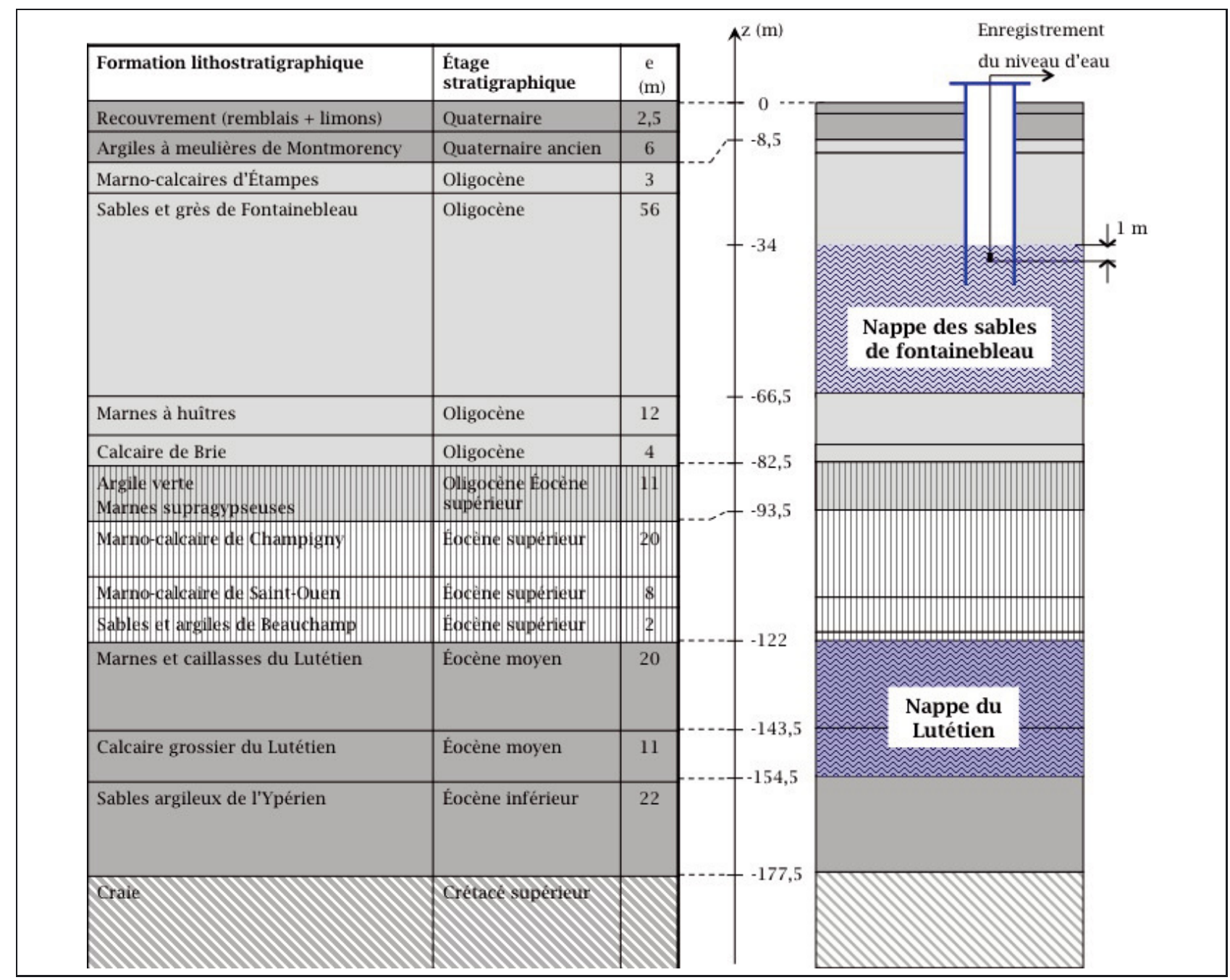

Fig. 1. - Coupe géologique synthétique à la verticale du site du LNE. La nappe des sables de Fontainebleau et la nappe du lutétien sont isolées l'une de l'autre. Le forage et la sonde piézométrique sont également représentés.

Bien que plusieurs approches aient été envisagées, deux semblent être en mesure d'atteindre des incertitudes relatives de $10^{-8}$, le projet Avogadro [7,8] et les expériences du type «balance du watt». La contribution française s'est tournée vers ce dernier type d'expérience en débutant en 2002 la réalisation d'une balance du watt [9].

Le principe de la balance du watt a été proposé par Brian Kibble en 1975 [10]. Il comporte deux phases, durant la phase statique, une puissance mécanique est obtenue à partir de la pesée d'une masse $m$, nécessitant une détermination absolue de l'accélération de la pesanteur au centre de gravité de cette masse avec une exactitude meilleure que $10^{-8}$. Il est donc nécessaire de disposer d'un gravimètre absolu. Cependant, cet instrument ne pouvant être placé sur la balance, il convient de transférer une mesure de $g$ obtenue à proximité de la balance, au centre de masse impliqué, sans en dégrader l'exactitude. Le LNE-SYRTE est en charge de cette détermination de l'accélération de la pesanteur $g$ et de son transfert.

Le présent article résume les travaux menés jusqu'à présent : du transfert à la mesure absolue de $g$ via le développement d'un nouvel instrument reposant sur les techniques de refroidissement d'atomes. Bien que l'accélération de la pesanteur s'exprime en $\mathrm{m} \cdot \mathrm{s}^{-2}$, les géophysiciens, principaux utilisateurs des signaux gravimétriques, continuent d'utiliser une unité de l'ancien système d'unités CGS : le gal ${ }^{1}$ (symbole Gal) [1] ainsi nommé en l'honneur de Galilée. Nous utilisons également cette unité dans l'article.

\section{Transfert de $g$ pour la balance du watt}

\subsection{Le site et les laboratoires}

L'expérience de balance du watt du LNE est menée sur le site de Trappes dans le bâtiment Maxwell. S'appuyant sur des études géologiques et hydrologiques du site [11], la construction du laboratoire a été adaptée aux conditions environnementales. Les premiers mètres du sol sont constitués de remblais inhomogènes et d'argiles à meulières, suivis de $56 \mathrm{~m}$ de sables de Fontainebleau (fig. 1). Sous ces sols, se succèdent des nappes d'argiles,

\footnotetext{
${ }^{1} 1 \mathrm{Gal}=1 \mathrm{~cm} \cdot \mathrm{s}^{-2}$ et donc $1 \mu \mathrm{Gal}=10^{-8} \mathrm{~m} \cdot \mathrm{s}^{-2} \approx 10^{-9}\langle\mathrm{~g}\rangle$.
} 


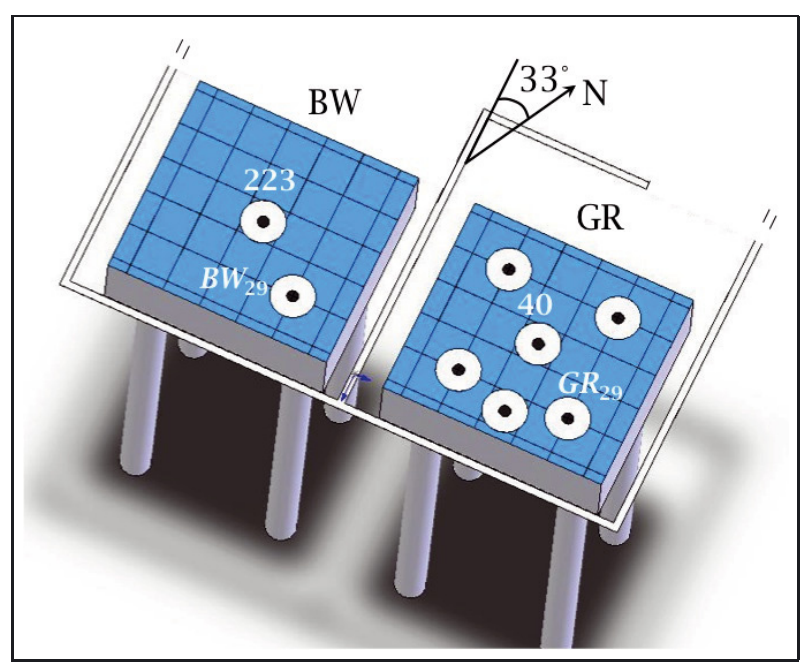

Fig. 2. - Dalles dans leurs salles respectives, à gauche, la salle pour la balance du watt notée « $\mathrm{BW}$ » de centre « 223 » et à droite, la salle pour la gravimétrie notée « GR » de centre « 40 ». Les massifs sont de tailles identiques : $6 \mathrm{~m} \times 5,5 \mathrm{~m} \times 2 \mathrm{~m}$. Les piliers soutenant les dalles ont une hauteur de $12 \mathrm{~m}$ et un diamètre de $80 \mathrm{~cm}$. Les murs en béton séparant les salles ont une hauteur de $6 \mathrm{~m}$ et une largeur de $20 \mathrm{~cm}$ Les points noirs représentent les stations d'accueil de gravimètres absolus.

de calcaires et de sables datant de l'oligocène, éocène et crétacé supérieur. La première nappe d'eau à la verticalité du site se trouve dans les sables de Fontainebleau à une profondeur d'environ $34 \mathrm{~m}$. L'épaisseur de cet aquifère saturé en eau est d'environ $34 \mathrm{~m}$ soit $60 \%$ de l'épaisseur totale des sables de Fontainebleau. Une seconde nappe se situe dans l'éocène moyen (lutétien) à 122 m. L'ypérien (éocène inférieur) n'est pas aquifère car très argileux. Dans le secteur de Trappes, la nappe du lutétien est captive sous les formations de l'éocène supérieur, marneuses et donc peu perméables. Ces deux nappes sont bien isolées l'une de l'autre. Un forage a été réalisé sur le site afin de suivre l'évolution de l'aquifère le moins profond avec un capteur piézométrique. Le second est pour l'instant observé à l'aide de données accessibles sur internet.

Pour garantir la stabilité du transfert de $g$ et pour maintenir les délicats alignements de la balance et du gravimètre, ces deux dispositifs doivent être placés sur des sols stables évitant des variations de hauteur et d'éventuelles variations de l'inclinaison. Pour cela, deux massifs identiques ont été construits dans deux salles voisines, l'une pour la balance du watt et l'autre pour la gravimétrie (fig. 2). Ainsi l'activité de gravimétrie peut être menée de façon indépendante, sans perturber le développement de la balance du watt. Ces massifs en béton de $6 \mathrm{~m} \times 5,5 \mathrm{~m} \times 2 \mathrm{~m}$ sont séparés d'une distance de $1,45 \mathrm{~m}$ et sont orientés avec un angle de $33^{\circ}$ par rapport au Nord. Ils sont soutenus par quatre pieds cylindriques d'une hauteur de $12 \mathrm{~m}$, également en béton, reposant sur les sables de Fontainebleau constituant une surface d'appui plus stable que les argiles. Pour éviter que ces argiles ne gonflent lors de pluies ou avec l'humidité et n'exercent une pression sur les massifs, un espace vide de $30 \mathrm{~cm}$ a été laissé sous les massifs et des drains ont

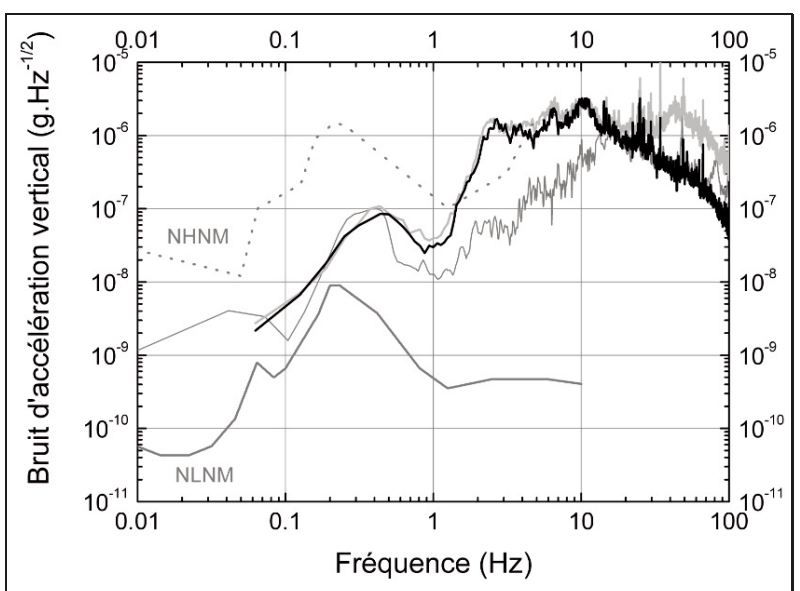

Fig. 3. - Spectres du bruit d'accélération mesurés avec un sismomètre bas bruit. La trace grise claire représente le spectre mesuré à côté des dalles et la trace noire représente le spectre mesuré sur les dalles. Le bruit de vibration est réduit après $20 \mathrm{~Hz}$. Le troisième spectre en gris foncé a été obtenu sur la dalle durant la nuit lorsque l'activité urbaine est interrompue. NHNM et NLNM représentent les bornes du modèle de Peterson (voir texte).

été réalisés. L'un des drains, situé à 2,5 $\mathrm{m}$ de profondeur entoure les massifs, le second entoure le bâtiment pour évacuer les écoulements de pluies. Les murs, représentés en blanc sur la figure 2, ont une hauteur de $6 \mathrm{~m}$ et une épaisseur de $20 \mathrm{~cm}$.

La figure 3 montre les spectres des vibrations verticales mesurés avec un sismomètre Guralp CMG-40T. Nous avons enregistré le spectre de vibrations de jour, sur et à côté des massifs. On voit clairement que le massif agit comme un filtre passe-bas avec une fréquence de coupure de $20 \mathrm{~Hz}$. On compare ces mesures avec des niveaux de bruits utilisés par les géophysiciens tels que les modèles de Peterson [12] : le nouveau modèle de bruit élevé NHNM (New High Noise Model) et le nouveau modèle bas bruit NLNM (New Low Noise Model). Ces modèles sont utilisés pour comparer les spectres de vibration. De $1,5 \mathrm{~Hz}$ à $10 \mathrm{~Hz}$, le bruit de vibration est au mieux confondu avec le NHNM, confirmant s'il était nécessaire, que le laboratoire se trouve dans une zone industrielle. Aux fréquences inférieures à $1,5 \mathrm{~Hz}$, le bruit d'accélération se situe entre le NHNM et le NLNM. Un spectre obtenu durant la nuit sur le massif est représenté en gris foncé sur la figure 3, il montre que le bruit de vibration peut baisser de plus d'un ordre de grandeur entre $2 \mathrm{~Hz}$ et $4 \mathrm{~Hz}$.

Les massifs sont recouverts d'un tapis plastifié conducteur de type linoleum. Ce revêtement a été enlevé au centre de chaque massif, en premier lieu aux emplacements dédiés à la balance du watt et au gravimètre absolu, puis à six autres endroits afin d'accueillir d'autres gravimètres pour effectuer des comparaisons. Ainsi, sur le massif de la salle de la balance du watt, nommée «BW », un gravimètre peut être installé en plus de la balance et sur le massif de la salle dédiée à la gravimétrie, nommée « GR », cinq gravimètres peuvent être installés en plus de 
notre gravimètre absolu. Ces stations de mesure sont marquées par des plots en aluminium anodisé scellés dans les massifs, et sont représentées par les points noirs sur la figure 2. Sur cette figure est également représenté un maillage établi sur chaque massif. Il est le même pour les deux massifs : 36 points répartis sur 6 lignes et 6 colonnes séparées d'une distance de $1 \mathrm{~m}$. Ils sont désignés par leur numéro respectif de 1 à 36 et précédés des deux lettres correspondant au massif. Les points centraux s'ajoutent à ce maillage et sont notés « $223 »$ pour la balance du watt et « 40 » pour le gravimètre absolu. Ce maillage de 74 points a été mis en place pour cartographier le laboratoire dans sa globalité.

Le gradient de gravité vertical étant de l'ordre de $3 \mu \mathrm{Gal} \cdot \mathrm{cm}^{-1}$, il est nécessaire de connaître les différences de hauteurs relatives avec une exactitude meilleure que $3 \mathrm{~mm}$ si l'on veut satisfaire notre objectif de déterminer $g$ avec une exactitude de $1 \mu \mathrm{Gal}$. Nous avons mesuré les différences de hauteurs relatives entre les différents points du maillage avec un niveau à eau. En moyenne, le massif BW est plus haut de $8 \mathrm{~mm}$ que le massif GR, la différence de hauteur entre les centres est de $15 \mathrm{~mm}$. L'incertitude de mesure est inférieure à $3 \mathrm{~mm}$.

\subsection{Gravimètre Scintrex CG5}

Le gravimètre relatif utilisé pour la cartographie est un « CG5 » commercialisé par la société Scintrex [13]. Il est dénommé «S105», en référence à son numéro de série, durant les campagnes de mesures relatives $\left(\mathrm{RGC}^{2}\right)[14,15]$ précédemment liées aux comparaisons internationales de gravimètres absolus $\left(\mathrm{ICAG}^{3}\right)$.

\subsubsection{Fonctionnement}

Les gravimètres Scintrex CG5 utilisent une masse suspendue à un ressort en quartz et un capteur capacitif de position. Cette masse $m$ est soumise à l'accélération de la pesanteur qui varie de $\delta g$, modifiant la longueur $d u$ ressort, de raideur $k$, de $\delta l$. On relie facilement ces quantités dans l'équation (1).

$$
\delta g=\frac{k}{m} \delta l+\delta k \frac{l}{m}
$$

$\delta k$ correspond à une variation éventuelle de la raideur du ressort dans le temps. Il est donc nécessaire de vérifier régulièrement et éventuellement d'ajuster la constante d'étalonnage de l'instrument.

Le ressort en quartz se trouve dans une enceinte à vide régulée en température. L'équilibre perturbé par un changement de pesanteur est détecté par un capteur capacitif contrôlant la position de la masse. La valeur relative de gravité est donnée par une contre-réaction forçant la masse à rester à la même position [16]. Un capteur mesure la température dans l'enceinte ce qui permet ensuite de corriger la gravité mesurée d'un effet thermique sur

\footnotetext{
${ }^{2}$ RGC : Relative Gravity Campaign.

3 ICAG : International Comparison of Absolute Gravimeters.
}

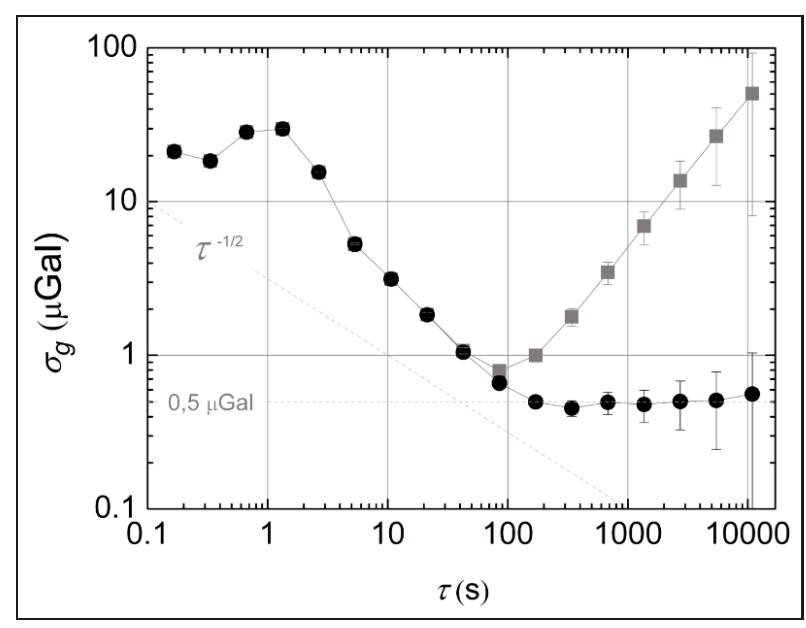

Fig. 4. - Sensibilités du gravimètre S105 obtenues sur le site GR représentées sous forme d'écarts type d'Allan. Les données correspondant aux carrés gris n'ont pas été corrigées des marées, contrairement aux points noirs.

le senseur. L'instrument est équipé de deux inclinomètres afin de corriger les mesures d'un éventuel défaut d'alignement vertical. Les écarts aux «zéros » et les sensibilités de ces inclinomètres sont étalonnés par l'opérateur. Il est également possible de corriger automatiquement les données d'une dérive linéaire du ressort mais nous forçons toujours cette correction à zéro et évaluons la dérive instrumentale lors de l'analyse des mesures. Enfin les mesures peuvent être corrigées de l'effet des marées luni-solaires, à l'aide d'un modèle intégré, calculé avec l'algorithme de Longman [17]. La fréquence de mesure du gravimètre CG5 est $6 \mathrm{~Hz}$ et il est possible de calculer la moyenne des données sur 256 s pour économiser la mémoire.

\subsubsection{Sensibilité}

Les performances du gravimètre S105 ont été éprouvées durant les premières années d'utilisation de l'instrument [18]. Nous rappelons ici brièvement quelle sensibilité de mesure ce type de gravimètre permet d'atteindre.

Le gravimètre CG5 dispose d'un mode d'acquisition continue des mesures. En plus des moyennes des données, les données brutes (échantillonnées à $6 \mathrm{~Hz}$ ) sont également stockées en mémoire. Ce mode nous a permis de caractériser la stabilité à court terme de l'instrument. Le choix de la durée d'acquisition maximale permise de 256 s s'impose lors de ces mesures afin de minimiser la durée des temps morts. La taille de la mémoire permet ainsi d'acquérir jusqu'à 12,5 h de données avec notre S105. Les gravimètres CG5 plus récents ont une mémoire trois fois plus importante permettant des acquisitions de $1,5 \mathrm{~d}$.

La stabilité obtenue à partir des signaux corrigés des variations de gravité, est exprimée par l'écart type d'Allan sur la figure 4. Pour les temps de mesure courts, inférieurs à $5 \mathrm{~s}$, la sensibilité est dégradée par le bruit sismique basse fréquence, dont l'amplitude est réduite par 
un filtre interne au gravimètre CG5. L'écart type d'Allan ne décroît pas en $\tau^{-1 / 2}$ comme en présence d'un bruit blanc. L'erreur statistique du gravimètre CG5 ne devrait donc pas être donnée, comme c'est le cas avec cet instrument, par la formule classique $e r r=s d \cdot N^{-1 / 2}$ avec $s d$ l'écart type et $N$ le nombre de mesures. Après $40 \mathrm{~s}$ de mesure, le gravimètre CG5 atteint une stabilité de $1 \mu \mathrm{Gal}$ qui continue de s'améliorer pour atteindre $0,8 \mu \mathrm{Gal}$ après $85 \mathrm{~s}$. Pour des temps de mesure supérieurs, la stabilité est dégradée par l'effet des marées. Après avoir corrigé les données brutes de cet effet, la stabilité s'améliore pour atteindre un palier à $0,5 \mu \mathrm{Gal}$ en $150 \mathrm{~s}$ d'intégration. Afin de réaliser des mesures avec une stabilité meilleure que $1 \mu \mathrm{Gal}$, nous avons choisi de moyenner $85 \mathrm{~s}$ les mesures effectuées lors des cartographies du site.

\subsection{Variations de l'accélération de la pesanteur}

L'accélération de la pesanteur varie dans le temps sous l'effet de l'attraction gravitationnelle des astres et des mouvements de masses au voisinage du point de mesure, tels que ceux des enveloppes fluides que sont l'atmosphère et les nappes phréatiques, pour ne citer que les plus connues. Il faut tenir compte de ces effets systématiques extérieurs pour déterminer une mesure absolue de $g$. Dans le cadre des mesures pour la balance du watt au LNE, il ne sera pas nécessaire d'effectuer ces corrections. Ces variations qui affectent le poids de la mesure pour la balance seront mesurées par le gravimètre atomique en temps réel et/ou avec un gravimètre supraconducteur. Cependant, dans le cadre de la caractérisation gravimétrique du site de Trappes et de l'étude du gravimètre atomique, il est important de bien modéliser ces effets afin de pouvoir comparer des mesures absolues déterminées à différents moments et de vérifier le bon fonctionnement du gravimètre absolu. Par la suite, la sensibilité de notre étalon pourrait permettre d'améliorer ces modèles voire même d'observer d'autres effets une fois la mesure corrigée des effets dominants présentés ici.

\subsubsection{Marées}

La déformation de la Terre due aux marées est une réponse élastique de son noyau aux accélérations produites par la lune, le soleil et dans une moindre ampleur, par les autres planètes [19]. Sous les latitudes européennes, les déformations journalières peuvent atteindre des amplitudes de $40 \mathrm{~cm}$ et un maximum de variation de gravité de $300 \mu \mathrm{Gal}$. Les marées dues au soleil représentent environ $46 \%$ des marées lunaires.

Les modèles ne peuvent prédire « exactement»le comportement général de l'effet des marées. Bien que la théorie statique permette de décrire l'influence des mouvements de la lune et du soleil, il est plus difficile de modéliser les corrections dues aux déplacements des océans. Il faut alors recourir à une théorie dynamique décomposant les océans en éléments dont les mouvements ont la même période que la force qui leur donne naissance et dont la somme des mouvements partiels élémentaires constitue le mouvement total. Plusieurs ouvrages traitent du développement mathématique de ce phénomène dont celui de Wenzel [20]. Les enregistrements de marées avec des gravimètres montrent d'importantes différences avec la théorie qui ne tient pas compte de la surcharge océanique [21]. L'amplitude des effets océaniques dépend de l'éloignement des côtes, il est de $3 \mu \mathrm{Gal}$ à $4 \mu \mathrm{Gal}$ à Trappes. Néanmoins, ce signal n'est pas en phase avec les marées terrestres.

Pour corriger les mesures gravimétriques de l'effet des marées dans son ensemble, on écrit cette correction comme une somme de termes périodiques :

$$
\Delta g_{\text {marées }}=-\sum \delta_{i} A_{i} \cos \left(\omega_{i} t+\phi_{i}+\Delta \phi_{i}\right)
$$

avec $\left(A_{i}, \omega_{i}, \phi_{i}\right)$ l'amplitude, la fréquence et la phase théoriques de l'onde $i$ provenant des modèles, et $\left(\delta_{i}, \Delta \phi_{i}\right)$ l'amplification et le déphasage observés pour cette même onde $i$. Le catalogue de potentiels de marées utilisé pour notre ajustement des coefficients est celui de Tamura [22] qui contient 1200 ondes (exactitude meilleure que $0,01 \mu \mathrm{Gal})$.

La première détermination de ces paramètres pour notre site de Trappes a été réalisée sur un enregistrement de 150 d du 13 décembre 2006 au 11 mai 2007 [23]. Il est possible d'ajouter à l' analyse, des enregistrements des paramètres environnementaux tels que la pression, la hauteur de nappes phréatiques, l'humidité dans le sol, etc. En 2007, nous ne disposions que des capteurs de pression. L'ajustement alors calculé, indiquant l'influence ou le facteur de corrélation de la pression sur notre mesure, plus communément nommée en anglais : barometric gravity admittance $\alpha$ est pour le gravimètre CG5 utilisé : $\alpha=$ $-0,27 \mu \mathrm{Gal} \cdot \mathrm{hPa}^{-1}$, proche de la valeur communément admise de $-0,3 \mu \mathrm{Gal} \cdot \mathrm{hPa}^{-1}$ [24] (voir paragraphe 2.3.2). L'incertitude du modèle est l'écart type du résidu entre les données enregistrées et la modélisation des variations obtenues, il est de $0,7 \mu \mathrm{Gal}$.

Deux autres déterminations ont été réalisées ensuite avec des enregistrements de 120 d obtenus avec deux gravimètres CG5 en parallèles sur le site : le gravimètre S105 du LNE-SYRTE et un gravimètre CG5 de l'Université du Luxembourg (CG5UL). Les deux analyses des paramètres de marées ont donné des résultats proches. L'écart type de l'ajustement obtenu avec le gravimètre CG5UL est de $1,2 \mu \mathrm{Gal}$ et l'admittance de $-0,16 \mu \mathrm{Gal} \cdot \mathrm{hPa}^{-1}$ contre encore $0,7 \mu \mathrm{Gal}$ avec le gravimètre $\mathrm{S} 105$ pour une admittance de $-0,26 \mu \mathrm{Gal} \cdot \mathrm{hPa}^{-1}$.

Finalement, nous utilisons le résultat de la dernière détermination effectuée sur 573 d de mesures. Les résultats sont représentés dans tableau 1. L'écart type de cet ajustement est encore de $0,7 \mu \mathrm{Gal}$ et l'admittance est de $-0,3 \mu \mathrm{Gal} \cdot \mathrm{hPa}^{-1}$.

Il ne semble pas possible d'obtenir une stabilité meilleure que $0,5 \mu \mathrm{Gal}$ sur le court terme et de $0,7 \mu \mathrm{Gal}$ sur le long terme avec le gravimètre S105 du laboratoire et donc d'obtenir des paramètres de marées permettant de modéliser ces variations de gravité à mieux que cette incertitude de 0,7 $\mu \mathrm{Gal}$. Cependant pour l'étude gravimétrique du site, cette incertitude est tout à fait satisfaisante. 
Tableau 1

Paramètres de marées obtenus à partir de 573 jours de mesure.

\begin{tabular}{|c|c|c|c|c|c|}
\hline Onde & $\begin{array}{c}\text { Amplitude } \\
(\boldsymbol{\mu G a l})\end{array}$ & $\boldsymbol{\delta}$ & $\boldsymbol{s d}$ & $\begin{array}{c}\boldsymbol{\Delta} \boldsymbol{\phi} \\
\mathbf{(}^{\circ}\end{array}$ & $\begin{array}{c}\boldsymbol{s} \boldsymbol{d} \\
\left({ }^{\circ}\right)\end{array}$ \\
\hline Q1 & 6,73 & 1,1414 & 0,0024 & $-0,621$ & 0,139 \\
\hline O1 & 35,25 & 1,1444 & 0,0005 & $-0,005$ & 0,027 \\
\hline M1 & 2,82 & 1,1648 & 0,0046 & $-0,051$ & 0,266 \\
\hline P1 & 16,46 & 1,1482 & 0,0012 & 0,450 & 0,068 \\
\hline K1 & 49,24 & 1,1368 & 0,0004 & 0,395 & 0,020 \\
\hline J1 & 2,79 & 1,1520 & 0,0059 & 0,199 & 0,341 \\
\hline OO1 & 1,53 & 1,1518 & 0,0066 & 0,085 & 0,379 \\
\hline 2N2 & 1,14 & 1,1447 & 0,0096 & 4,506 & 0,553 \\
\hline N2 & 7,35 & 1,1750 & 0,0019 & 4,036 & 0,109 \\
\hline M2 & 38,79 & 1,1880 & 0,0004 & 3,333 & 0,021 \\
\hline L2 & 1,11 & 1,2061 & 0,0136 & 1,101 & 0,777 \\
\hline S2 & 18,20 & 1,1980 & 0,0008 & 1,345 & 0,045 \\
\hline K2 & 4,99 & 1,2075 & 0,0021 & 1,523 & 0,123 \\
\hline M3 & 0,45 & 1,0504 & 0,0229 & $-0,261$ & 1,314 \\
\hline M4 & 0,17 & 3,3461 & 1,5152 & $-77,747$ & 86,816 \\
\hline
\end{tabular}

\subsubsection{Pression atmosphérique}

Les variations de gravité générées par les variations de la masse de l'atmosphère peuvent être séparées en deux effets : l'effet direct de l'attraction de la masse d'air et l'effet indirect de charge de la masse d'air sur la croûte terrestre et les océans. Les déformations de la surface peuvent en effet atteindre $1 \mathrm{~cm}$. Les variations de l'accélération de la pesanteur sont par conséquent corrélées avec les variations de la pression atmosphérique, avec une admittance de $-0,3 \mu \mathrm{Gal} \cdot \mathrm{hPa}^{-1}$ à $-0,4 \mu \mathrm{Gal} \cdot \mathrm{hPa}^{-1}$ selon les régions, les conditions météorologiques et la proximité de la mer. Durant les mesures relatives ou absolues, il est nécessaire de mesurer les variations de pression atmosphérique pour corriger les mesures. La résolution 9 de l'IAG ${ }^{4}$ recommande d'utiliser une admittance de $-0,3 \mu \mathrm{Gal} \cdot \mathrm{hPa}^{-1}$ comme moyenne globale par défaut [25]. La correction de la mesure de pesanteur est basée sur le «U.S Standard Atmosphere », 1976 pris comme référence de modèle atmosphérique et s'écrit :

$$
\Delta g_{\text {air }}=-\alpha\left(P-P_{\mathrm{n}}\right),
$$

avec $P$ la pression atmosphérique et $P_{\mathrm{n}}$ la pression atmosphérique nominale ainsi définie :

$$
P_{\mathrm{n}}=1013,25 \times\left(1-\frac{0,0065 \times h}{288,15}\right)^{5,2559},
$$

avec $h$ la hauteur de mesure par rapport au niveau des mers. Sur le site GR de Trappes, $P_{\mathrm{n}}=992,99 \mathrm{hPa}$.

Si la pression varie de $30 \mathrm{hPa}$, le coefficient $\alpha$ doit être connu avec une incertitude meilleure que $5 \%$ pour obtenir des corrections avec des incertitudes de 0,5 $\mu \mathrm{Gal}$. Ce paramètre pris usuellement comme constant n'a en fait aucune raison de l'être. Il dépend notamment des nuages et de ce qu'ils contiennent.

\footnotetext{
${ }^{4}$ IAG : International Association of Geodesy.
}

\subsubsection{Mouvement du pôle}

L'orientation de l'axe de rotation de la Terre n'est pas fixe par rapport à la croûte terrestre, si bien que la position des pôles peut ainsi varier de quelques mètres par an. Ces variations modifient l'accélération de la pesanteur au point de notre mesure par variation de l'accélération centrifuge. L'IERS ${ }^{5}$, service international qui détermine la vitesse de rotation de la Terre, fournit toutes les semaines les coordonnées $x_{\mathrm{p}}$ et $y_{\mathrm{p}}$ du pôle Nord par rapport au pôle de référence. Ces coordonnées sont définies dans un plan tangent au pôle avec l'axe $x$ dans la direction du méridien de Greenwhich et l'axe $y$ pointant vers le méridien $90^{\circ} \mathrm{O}$. Le mouvement du pôle est principalement composé de deux périodes de 365 et 435 jours et d'une dérive irrégulière $[26,27]$. La correction de la mesure de gravité est donnée par [28] :

$$
\Delta g_{\text {pôle }}=-\delta_{\text {pol }} \omega^{2} r \sin 2 \phi\left(x_{\mathrm{p}} \cos \lambda-y_{\mathrm{p}} \sin \lambda\right) \text {, }
$$

avec $\omega$ la vitesse de rotation de la Terre, $r$ le rayon moyen de la Terre et $(\lambda, \phi)$ les latitude et longitude du point de mesure. Le facteur d'amplitude $\delta_{\text {pol }}=1,16$ prend en compte la réponse élastique de la Terre. L'erreur sur cette correction est inférieure à $0,1 \mu \mathrm{Gal}$, grâce à l'exactitude des coordonnées fournies par l'IERS $\left( \pm 0,0003^{\prime \prime}\right)$.

La variation de la correction à apporter aux mesures n'est pas assez rapide pour qu'il soit nécessaire d'en tenir compte dans nos caractérisations gravimétriques du site. Par contre, il faut en tenir compte si on souhaite fournir une mesure absolue de $g$ et comparer cette mesure à une mesure plus ancienne correspondant à une position du pôle différente.

\subsubsection{Nappes phréatiques}

Comme toute variation de masse environnant la station, la variation du niveau des nappes phréatiques modifie l'accélération de la pesanteur [29,30]. Nous avons placé un capteur piézométrique dans un forage afin de suivre l'évolution du niveau de la nappe des sables de Fontainebleau (paragraphe 2.1). Ce capteur est étalonné afin de mesurer des variations du niveau d'eau d'une amplitude de $2 \mathrm{~m}$ sur sa plage de mesure de $4 \mathrm{~mA}$ à $20 \mathrm{~mA}$. Il est placé à $1 \mathrm{~m}$ sous le niveau de la nappe. Ces capteurs piézométriques déduisent la hauteur d'immersion dans l'eau de la pression mesurée dans l'eau. Un « capillaire » remontant jusqu'à la surface permet de soustraire la pression atmosphérique à la mesure.

Le calcul du changement de gravité engendré par un espace vide de dimensions infinies se remplissant d'eau, donne une dépendance de $0,4 \mu \mathrm{Gal} \cdot \mathrm{cm}^{-1}$. L'espace impliqué ici est composé de sables et de grès de Fontainebleau de porosité située entre $15 \%$ et $25 \%$ ce qui modère l'effet sur les variations de gravité. Une variation de $10 \mathrm{~cm}$ de la nappe phréatique correspondrait donc ici à une variation de la maximale de gravité de $1 \mu \mathrm{Gal}$.

\footnotetext{
${ }^{5}$ IERS : International Earth Rotation and reference Systems service.
} 
Nous avons jusqu'à présent observé des variations du niveau de la nappe de $10 \mathrm{~cm}$ autour de $1 \mathrm{~m}$ d'immersion de la sonde mais nous n'avons pas pu corréler ces variations avec les variations de pesanteur mesurées par les gravimètres à ressort et atomique. Des études plus approfondies avec le gravimètre supraconducteur permettront peut-être d'observer de telles corrélations.

\subsection{Suivi des variations de l'accélération de la pesanteur}

Les modèles ne prenant pas en compte toutes les sources de variation de l'accélération de la pesanteur, il est plus judicieux de mesurer ces variations en continu. Comme expliqué précédemment, le gravimètre Scintrex CG5 peut enregistrer les variations de gravité mais sa forte dérive nous empêche de connaître autour de quelle valeur absolue ces variations se produisent.

Le gravimètre atomique permettra de mesurer les variations de $g$ durant les sessions de mesure de la balance du watt. Par ailleurs, nous disposons depuis 2013 d'un nouveau gravimètre relatif supraconducteur (modèle iGrav de la société GWR [31]). Le principe de mesure est similaire au gravimètre CG5, mais la masse est une sphère supraconductrice et le « ressort » est un champ magnétique très stable généré par des bobines supraconductrices. Le courant nécessaire pour faire léviter la sphère et la maintenir à la même position dépend de l'accélération de la pesanteur. L'ensemble est maintenu dans un cryostat à la température de l'hélium liquide. Bien que ce gravimètre ait été conçu pour être déplaçable, il est généralement utilisé pour observer les variations de pesanteur sur un site fixe, dans des observatoires gravimétriques. Comme le gravimètre CG5, son facteur d'échelle est à étalonner régulièrement. La fréquence de mesure de cet instrument est de $12 \mathrm{~Hz}$, et il donne accès à une valeur de $g$ toutes les secondes. Il sera un outil précieux pour délivrer un signal continu à la balance du watt, contrôlé par le gravimètre atomique absolu. Il permettra de plus de vérifier le fonctionnement correct du gravimètre atomique entre différentes campagnes de mesures ou autres interventions et interruption des mesures.

Les gravimètres supraconducteurs ont un niveau de bruit très faible et permettent d'obtenir des sensibilités inférieures à $1 \mu \mathrm{Gal}$ en quelques secondes comme indiqué sur la figure 5. En particulier, cet instrument peut atteindre des sensibilités de l'ordre de $10 \mathrm{nGal}$ au bout de 400 s de mesures. Sur des temps plus longs, la stabilité de la mesure se dégrade à cause des variations de gravité liées aux marées terrestres. Le modèle de marée utilisé n'ayant pas une précision suffisante pour les capacités de mesure du gravimètre supraconducteur, une nouvelle détermination des paramètres de marées à l'aide des signaux du gravimètre iGrav est en cours.

\subsection{Cartographie gravimétrique des laboratoires}

Cette partie est consacrée aux études que nous avons effectuées afin de transférer une valeur absolue de $g$ mesurée en un point, en n'importe quel autre point des deux

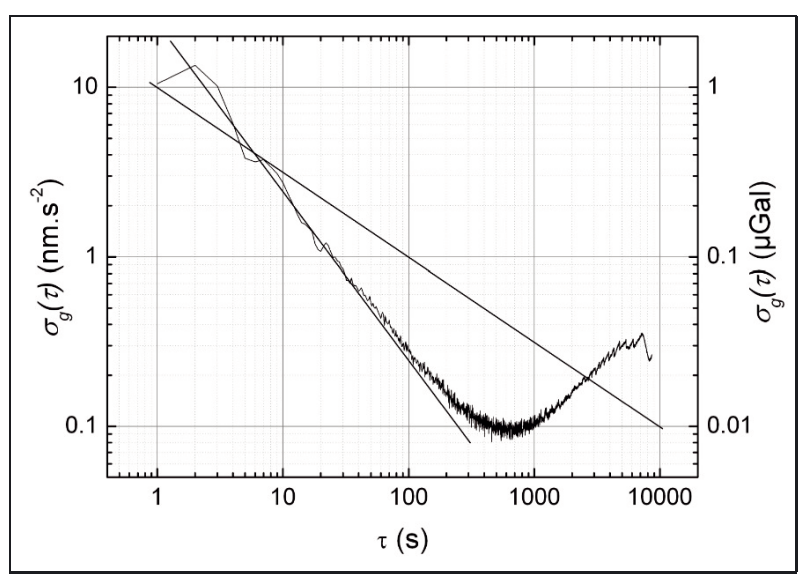

Fig. 5. - Sensibilité du gravimètre iGrav-005 obtenue sur le site GR représentée sous forme de l'écart type d'Allan sur une période calme en mars 2013. Les droites de pente $\tau^{-1 / 2}$ et $\tau^{-1}$ sont indiquées pour comparer à la variation de la sensibilité du gravimètre $\mathrm{S} 105$ de la figure 4.

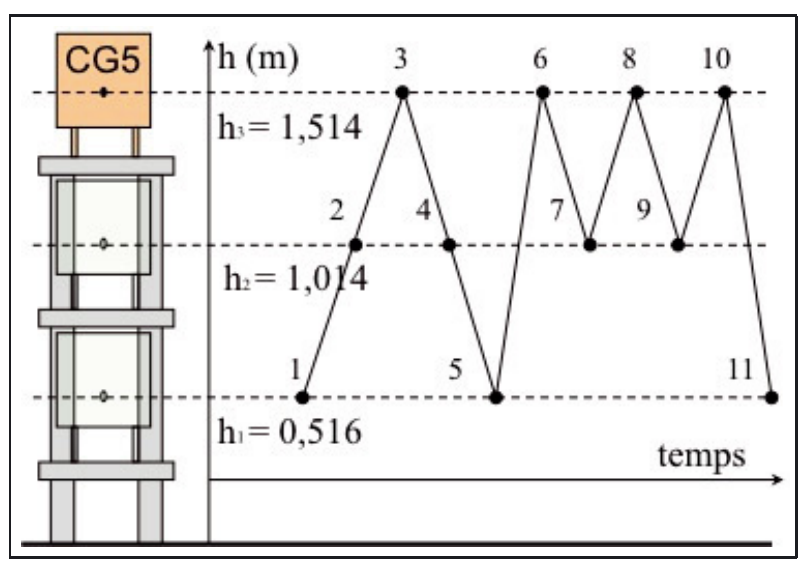

Fig. 6. - Protocole de mesure utilisé pour déterminer les gradients verticaux de gravité (vgg). Il comporte 11 positions de mesure à trois hauteurs différentes.

massifs. Un modèle du champ de gravité dans le laboratoire a été réalisé permettant de connaître les différences entre tous les points des laboratoires dans un volume de $50 \mathrm{~m}^{3}$. Il est ainsi possible de comparer deux gravimètres sans avoir besoin de les déplacer mais surtout, de transférer la mesure de $g$ du gravimètre atomique au centre de masse de l'étalon impliqué dans la balance du watt. Ce paragraphe reprend les éléments d'un article publié dans Metrologia [23].

\subsubsection{Gradients verticaux de gravité (vgg)}

Les gradients verticaux de gravité, notés $v g g^{6}$ ont été déterminés en mesurant la gravité à trois hauteurs. La figure 6 détaille le protocole mis en place pour la détermination des $v g g$. Il consiste à mesurer la gravité à 11 positions successives. À chaque position, deux à trois mesures de $85 \mathrm{~s}$ sont effectuées selon le bruit sismique. Les mesures sont corrigées d'une dérive résiduelle commune

\footnotetext{
${ }^{6}$ vgg: vertical gravity gradient.
} 
qui est la moyenne pondérée des trois dérives individuelles calculées à chaque hauteur. La valeur de la gravité à chaque hauteur est la moyenne pondérée des mesures à cette hauteur. La pondération choisie est l'inverse des écarts types au carré $\left(1 / s d^{2}\right)$. L'écart type de la moyenne des mesures à une même hauteur permet de pondérer par la suite le poids des trois valeurs de gravité lors du meilleur ajustement de la courbe du $v g g$.

Si on prend en compte la hauteur du senseur dans le gravimètre CG5, l'empilement des différents trépieds nous permet de mesurer la gravité aux hauteurs effectives de 51,6 cm, 101,4 cm et 151,4 cm (fig. 6). Nous avons choisi ces hauteurs pour encadrer la hauteur de $1 \mathrm{~m}$, hauteur initialement envisagée pour la masse impliquée dans la balance du watt.

Lors des différents essais ayant aboutis à ce protocole de mesure, des mesures à une quatrième hauteur de $26 \mathrm{~cm}$ ont été ajoutées. Cette hauteur était obtenue en posant le tripode du gravimètre CG5 directement sur le sol. Le but de ces mesures était de détecter une éventuelle non-linéarité du vgg proche du sol en disposant d'un point supplémentaire lors de la recherche de la courbe du meilleur ajustement du vgg avec un polynôme de degré 2 . Les termes non linéaires ne sont pas significatifs sur trois ou quatre points. Chaque station de mesure supplémentaire rallonge bien entendu le temps nécessaire à la détermination d'un vgg. De plus, comme nous cherchons à déterminer le $v g g$ à une hauteur proche de $1 \mathrm{~m}$, cette quatrième position a rapidement été supprimée du protocole en cours d'élaboration. Dès lors, le vgg a été considéré comme linéaire entre $0,5 \mathrm{~m}$ et $1,5 \mathrm{~m}$ ce qui s'est par la suite confirmé lors des modélisations (paragraphe 2.6).

Durant plusieurs mois, les vgg aux 70 points accessibles sur les massifs ont été déterminés. Certains l'ont été à plusieurs reprises afin d'évaluer la stabilité des déterminations. Ainsi 8 déterminations ont été effectuées au point « 223 » durant 3 mois. L'écart type de la distribution de ces 8 déterminations et de $1,0 \mu \mathrm{Gal} \cdot \mathrm{m}^{-1}$.

À partir de ces différents tests et résultats, en prenant en compte l'influence de la dérive résiduelle $\left(0,2 \mu \mathrm{Gal} \cdot \mathrm{m}^{-1}\right)$ et l'incertitude sur la détermination de la courbe linéaire de meilleur ajustement $\left(0,5 \mu \mathrm{Gal} \cdot \mathrm{m}^{-1}\right), l^{\prime}$ incertitude sur la détermination du $\mathrm{vgg}$ est de $0,5 \mu \mathrm{Gal} \cdot \mathrm{m}^{-1}$. En considérant l'écart type pour les mesures répétées, on trouve une incertitude sur la détermination légèrement supérieure, de $1,0 \mu \mathrm{Gal} \cdot \mathrm{m}^{-1}$. Avec une telle incertitude et à la hauteur de détermination de $1 \mathrm{~m}$, il n'est pas possible de détecter une non-linéarité du vgg dans notre laboratoire. Depuis les premières mesures en 2005, plusieurs points ont été revisités, les vgg obtenus ne diffèrent pas de plus de $1,0 \mu \mathrm{Gal} \cdot \mathrm{m}^{-1}$.

La figure 7 représente la cartographie des $v g g$ sur les deux massifs.

\subsubsection{Cartographie horizontale}

Chaque massif a été cartographié indépendamment et de manière identique avec le point central comme station

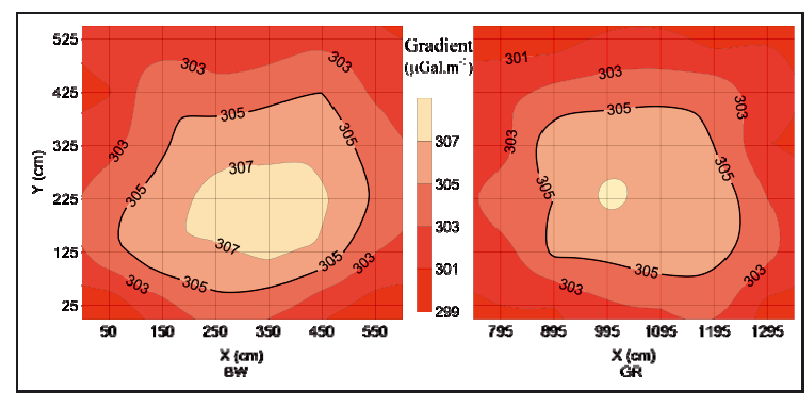

Fig. 7. - Cartographie des vgg sur les deux massifs.

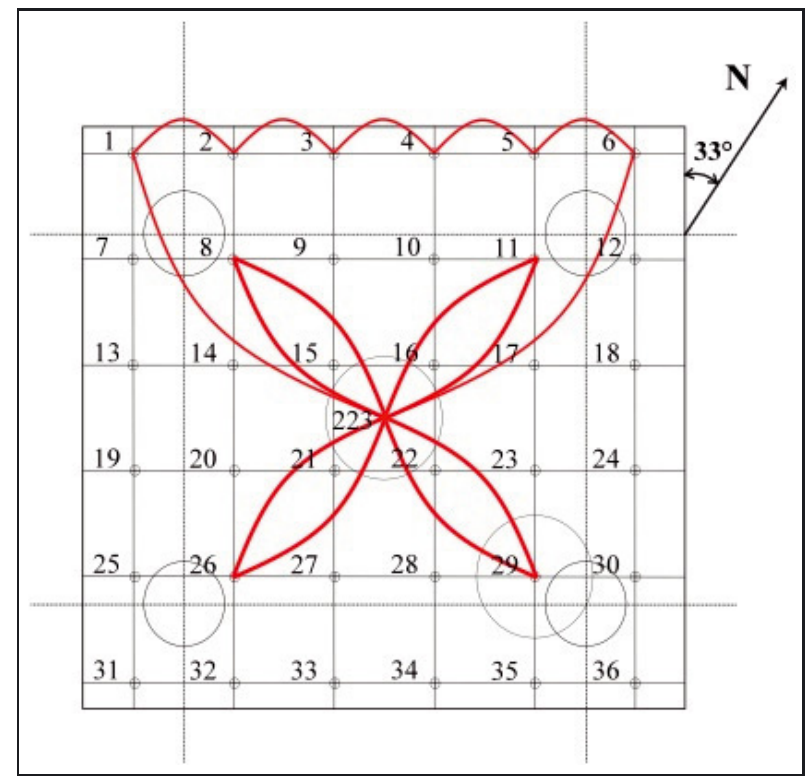

Fig. 8. - Protocole de mesure des différences de gravité relative représenté ici sur le massif BW. Les quatre cercles identiques représentent les pieds de $12 \mathrm{~m}$ soutenant la dalle.

de référence. Les mesures ont été effectuées à 51,6 cm du sol à l'aide du petit tripode (fig. 6). Le protocole de mesure est représenté sur la figure 8 . Il consiste à effectuer une étoile à quatre bras, centrée sur la station de référence puis à visiter les six points de chaque ligne horizontale en repassant par la station de référence entre chaque ligne et enfin l'étoile est refaite pour clore les mesures. Ce protocole a été mis en place afin de déterminer au mieux la dérive résiduelle [26]. Deux mesures successives de $85 \mathrm{~s}$ sont effectuées à chaque point. La dérive est déterminée entre deux passages successifs sur la station de référence et les différences de gravité sont calculées relativement au point central. Le protocole permet d'être positionné à trois instants différents aux quatre points « $8 », \ll 11 »$, « $26 »$ et « $29 »$ pour chaque massif. On a ainsi obtenu trois différences de gravité relativement aux points centraux pour ces huit points. L'écart type de ces trois valeurs varie de $0,4 \mu \mathrm{Gal}$ à $2,3 \mu \mathrm{Gal}$ avec une moyenne de $0,9 \mu \mathrm{Gal}$ pour le massif $\mathrm{BW}$ et $1,7 \mu \mathrm{Gal}$ pour le massif GR, valeurs que nous avons prises comme incertitude de mesure pour chaque cartographie. Les variations de gravité obtenues varient jusqu'à $20 \mu \mathrm{Gal}$; ce qui est bien supérieur aux incertitudes de mesure. 


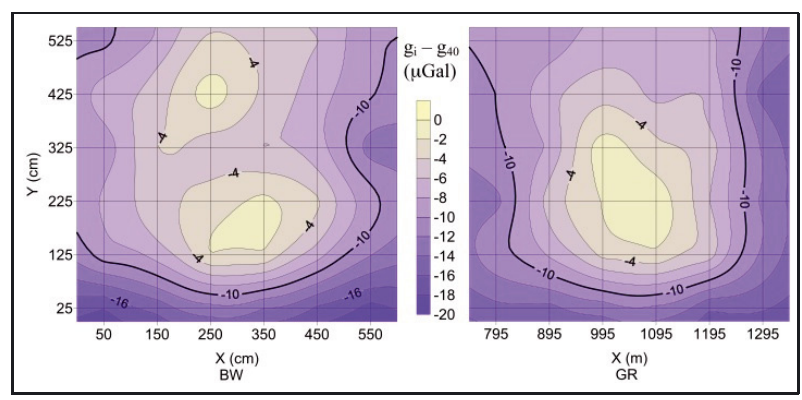

Fig. 9. - Champ de gravité sur les massifs après correction des effets de latitude et de hauteur, relativement au point « 40 », centre du massif GR.

Durant six mois, plusieurs liens entre différents points des deux massifs ont été répétés, dont six entre les deux points centraux, références de chaque massif. Ces différences obtenues sont stables : l'écart type des différences entre les deux points centraux « 223 » et « 40 » est inférieur à $0,2 \mu \mathrm{Gal}$. La station de référence choisie pour toutes les différences de gravité relative est le point « 40 » sur le massif GR, station d'accueil du gravimètre atomique. À partir de l'écart de gravité mesuré entre les deux centres, on cartographie les différences de gravité des deux massifs relativement au point $« 40 »$.

Les massifs sont orientés avec un angle de $33^{\circ}$ par rapport au Nord (fig. 2 et 8). En prenant en compte la Formule de Gravité Internationale applicable dans le système GRS $80^{7}$ ainsi que la position des massifs (48 $45^{\prime} 38,58^{\prime \prime} \mathrm{N}, 1^{\circ} 59^{\prime} 3,8^{\prime \prime}$ (WGS84)), on calcule que la gravité varie de $0,808 \mu \mathrm{Gal} \cdot \mathrm{m}^{-1}$ en direction du Nord. Ainsi, compte tenu de l'orientation des massifs, d'un point situé à un coin d'un massif (point « 6 ») à celui situé au coin opposé (point «31») la différence de gravité attendue, due à la différence de latitude est de 5,6 $\mu \mathrm{Gal}$ et de $8,9 \mu \mathrm{Gal}$ entre les points $\mathrm{BW}_{31}$ et $\mathrm{GR}_{6}$. Nous avons corrigé les différences de gravité mesurées relativement au point « 40 » de l'effet de la latitude. Nous avons également corrigé ces différences de l'effet des différences de hauteur avec le point « 40 » en utilisant les vgg. Nous avons ainsi pu tracer sur la figure 9 les différences de gravité relatives au point « 40 » ne montrant que des différences dues à la répartition des masses locales.

Compte tenu de (i) l'incertitude sur la différence de hauteur entre les points donnant la plus grande valeur de $v g g$ de $0,9 \mu \mathrm{Gal}$; (ii) de l'incertitude sur le $v g g$ de $1,0 \mu \mathrm{Gal}$; (iii) de l'incertitude sur le lien entre les deux centres de $0,2 \mu \mathrm{Gal}$ et (iv) de l'incertitude des cartographies des massifs de 0,9 $\mu \mathrm{Gal}$ et 1,7 $\mu \mathrm{Gal}$, l'incertitude de la cartographie relativement au point « $40 »$ du massifs BW est de 1,6 $\mu \mathrm{Gal}$ et de 2,2 $\mu \mathrm{Gal}$ pour le massif GR.

\subsection{Modélisation des variations de gravité dues au laboratoire}

Nous avons considéré que les anomalies de gravité résiduelles (fig. 9) sont dues à l'attraction des

\footnotetext{
${ }^{7}$ GRS80 : Geoid Reference System 1980.
}

masses environnantes. Plusieurs éléments sont susceptibles d'avoir un impact sur notre détermination du transfert de $g$. Connaissant, par construction, la géométrie de ces différents éléments, leurs densités ont été prises comme paramètres libres à ajuster. Nous avons considéré ces densités uniformes pour chaque élément. La modélisation de ces éléments connus repose sur un algorithme d'attraction d'éléments parallélépipédiques [32]. Nous avons ensuite minimisé le résidu de la différence entre le champ calculé et les mesures observées à $52 \mathrm{~cm}$ du sol et pour les $v g g$. La minimisation du résidu s'effectue en ajustant les densités et en ajoutant des éléments, si bien qu'au final, la modélisation comprend : les massifs, les pieds des massifs, les vides sous les dalles et les murs entourant les laboratoires. Le meilleur ajustement est obtenu pour des densités des massifs BW et GR de 2,2 et 2,3, valeurs proches des densités mesurées, respectivement de 2,23 et 2,25. Ces mesures ont été effectuées lors du carottage des dalles pour la mise en place des plots en aluminium anodisé repérant les points dédiés aux gravimètres absolus.

L'écart type de la distribution des résidus de $v g g$ est de $1,7 \mu \mathrm{Gal} \cdot \mathrm{m}^{-1}$ sur l'ensemble des massifs et de $0,9 \mu \mathrm{Gal} \cdot \mathrm{m}^{-1}$ sur les $1 \mathrm{~m}^{2}$ centraux. Nous avons par ailleurs calculé les vgg à différentes hauteurs du sol. Les résultats ont confirmé la linéarité des vgg comme précédemment mentionné au paragraphe 2.5.1. Concernant les résidus de la cartographie horizontale, l'écart type est de 2,8 $\mu \mathrm{Gal}$ sur l'ensemble des dalles et de 1,8 $\mu \mathrm{Gal}$ sur les $1 \mathrm{~m}^{2}$ centraux. Ces incertitudes sont essentiellement dues aux incertitudes de nos cartographies de 1,6 $\mu \mathrm{Gal}$ et de $2,2 \mu \mathrm{Gal}$.

Les résultats de cette modélisation ont été confirmés par la suite par comparaison à une seconde modélisation indépendante basée sur une technique mise en place en collaboration avec Giancarlo D'Agostino de l'INRIM dans le cadre du projet européen le $\mathrm{JRP}^{8}$ e-Mass [33]. Cette dernière technique a depuis été utilisée pour modéliser l'effet de l'attraction propre du gravimètre atomique et de la balance.

\subsection{Comparaison avec des mesures absolues}

Comme nous le montrons au paragraphe 4, plusieurs comparaisons de gravimètres absolus ont eu lieu sur le site de Trappes. La première a eu lieu en 2006, alors que les deux massifs étaient inoccupés. Elle impliquait des gravimètres de type $\mathrm{FG}^{9}$. Les points « 40 » et $\mathrm{GR}_{29}$ sur le massif GR et « 223 » et $\mathrm{BW}_{29}$ sur le massif $\mathrm{BW}$ ont ainsi été mesurés par trois gravimètres FG5 [36]. Les résultats sont représentés sur la figure 18. Les points « 29 » des deux massifs ont été mesurés deux fois à des jours différents par le gravimètre FG5\#215. Les résultats montrent

\footnotetext{
8 JRP : Joint Research Project de l'EMRP; l'EMRP est cofinancé par les pays participants à l'EMRP dans le cadre d'Euramet et par l'Union Européenne.

${ }^{9}$ FG5 : Gravimètre commercialisé par la société Micro-g Lacoste et utilisant la chute libre d'un miroir observée à l'aide d'un laser [34, 35].
} 


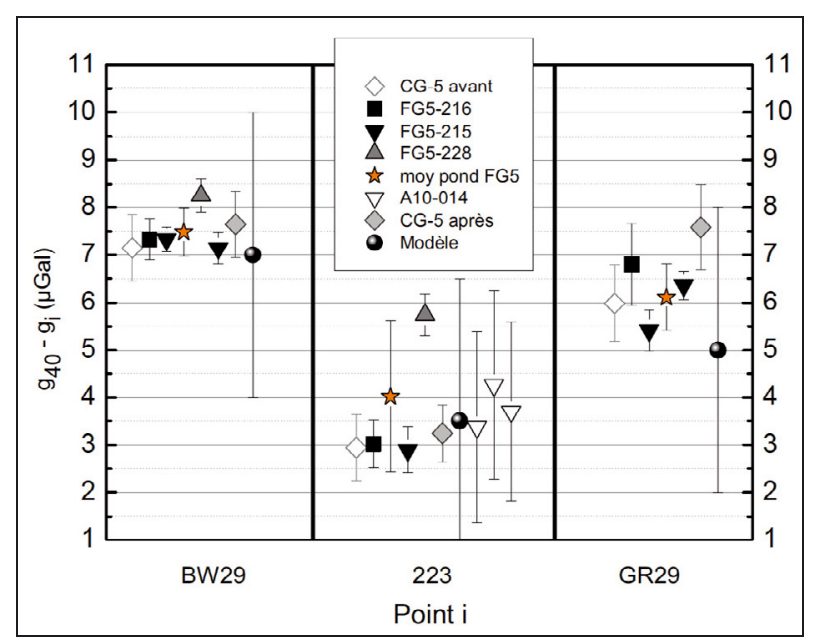

Fig. 10. - Différences de gravité relatives au point « 40 » obtenues à partir des mesures absolues de la comparaison de 2006. Les losanges représentent les mesures relatives obtenues avant et après la comparaison avec le gravimètre CG5 et les ronds les mêmes différences obtenues avec le modèle de variation de gravité.

une excellente répétabilité des mesures. Bien que l'on relève une différence entre les différents instruments allant jusque $10 \mu \mathrm{Gal}$, l'excellente répétabilité des mesures permet néanmoins de calculer les différences de gravité relativement au point « 40 ». Les résultats sont présentés sur la figure 10 et sont comparés avec des mesures effectuées avec le gravimètre CG5 avant et après les mesures absolues. Afin de mesurer à une hauteur proche de $122 \mathrm{~cm}$, nous avons utilisé des tripodes permettant d'effectuer des mesures à $128 \mathrm{~cm}$. Les valeurs ont ensuite été transférées à la hauteur de $122 \mathrm{~cm}$ avec les $v g g$. Les résultats relatifs obtenus avec le gravimètre CG5 sont en accord avec la moyenne pondérée des différences relatives obtenues avec les gravimètres FG5. Cette comparaison à des mesures relatives obtenues avec des gravimètres absolus confirme la validité de protocoles de mesure et de traitement des mesures avec notre gravimètre CG5 ainsi que celle du modèle.

\subsection{Effet de la balance sur la gravité}

La balance du watt est elle-même un corps massique proche de la masse impliquée dans la mesure. Son effet doit être pris en compte dans le transfert final de la valeur absolue de $g$. Basé sur la technique de modélisation mise en place pour calculer l'effet de gravité propre du gravimètre atomique [33], la balance a été modélisée pour déterminer son effet d'attraction.

Bien que les interféromètres finaux (qui permettent le contrôle du déplacement de la bobine dans la balance) ne soient pas entièrement réalisés, les autres éléments de la balance ont été modélisés et leurs effets sur la pesanteur calculés sur une distance de $20 \mathrm{~cm}$. Ainsi l'effet global peut être connu quelle que soit la hauteur de pesée choisie à l'avenir. La hauteur actuellement prévue pour le centre de gravité de la masse impliquée dans l'expérience

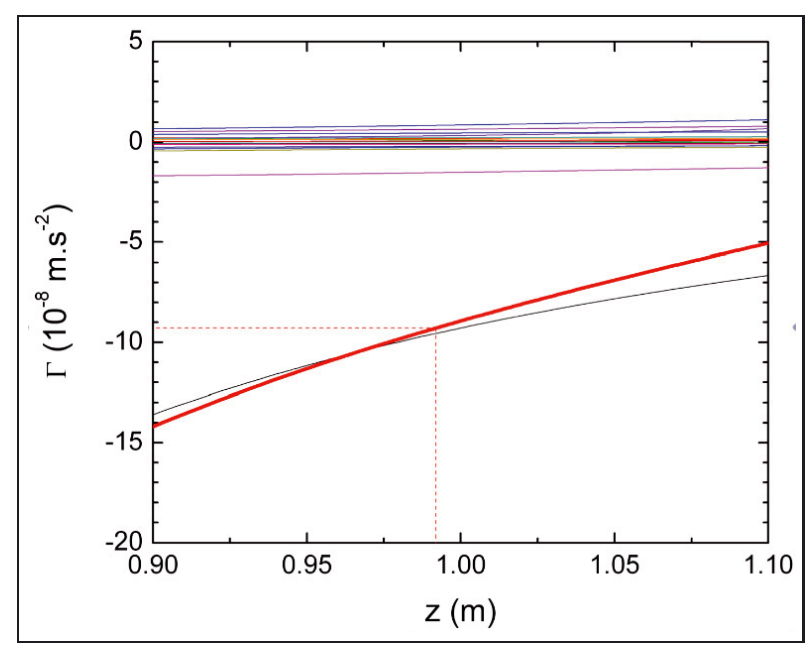

Fig. 11. - Effet d'attraction $(\Gamma)$ des différents éléments composants la balance du watt (traits fins) en fonction de la hauteur par rapport au sol. La somme, représentant l'effet d'attraction total, est indiquée en trait épais. La position du centre de masse de la masse impliquée dans l'expérience est indiquée en pointillés $(0,992 \mathrm{~m})$, l'attraction est de $9,3 \times 10^{-8} \mathrm{~m} \cdot \mathrm{s}^{-2}$ vers le sol.

de la balance du watt est de 99,2 cm. La figure 11 représente l'effet d'attraction des différents éléments composant la balance et l'effet total. L'aimant $(350 \mathrm{~kg})$ situé juste en dessous de la masse domine l'effet total de l'expérience, respectivement $9,6 \times 10^{-8} \mathrm{~m} \cdot \mathrm{s}^{-2}$ (ou 9,6 $\mu \mathrm{Gal}$ ) et $9,3 \times 10^{-8} \mathrm{~m} \cdot \mathrm{s}^{-2}$. L'incertitude associée est étendue à $0,1 \times 10^{-8} \mathrm{~m} \cdot \mathrm{s}^{-2}[33]$.

\section{Détermination absolue de $g$}

Chaque expérience de balance du watt menée dans les différents instituts nationaux de métrologie comporte ses propres spécificités, la distinguant des autres [37,38]. Cependant pour obtenir une mesure absolue de l'attraction de gravité, tous les laboratoires utilisent un gravimètre à coin de cube de type FG5 commercialisé par la société Micro-gLaCoste Inc. [35], à l'exception du LNE qui a choisi de développer et d'utiliser un gravimètre atomique.

Le projet de gravimètre atomique du LNE-SYRTE a débuté à l'Observatoire de Paris, dans le cadre du projet «Balance du watt». Un prototype a tout d'abord été réalisé afin d'effectuer des premières mesures [39] et d'éprouver sa sensibilité [40] puis d'évaluer les effets systématiques [41]. Parallèlement, un gravimètre plus abouti a été conçu sur la base de l'expérience accumulée avec ce prototype.

\subsection{Principe de la mesure}

Pour les deux dispositifs, le principe de fonctionnement et de mesure est le même, et la théorie a été développée dans les divers travaux de recherche de l'équipe et notamment de thèses de doctorat disponibles en accès libre [42]. Nous rappelons ici brièvement les éléments nécessaires à la compréhension de l'article. 


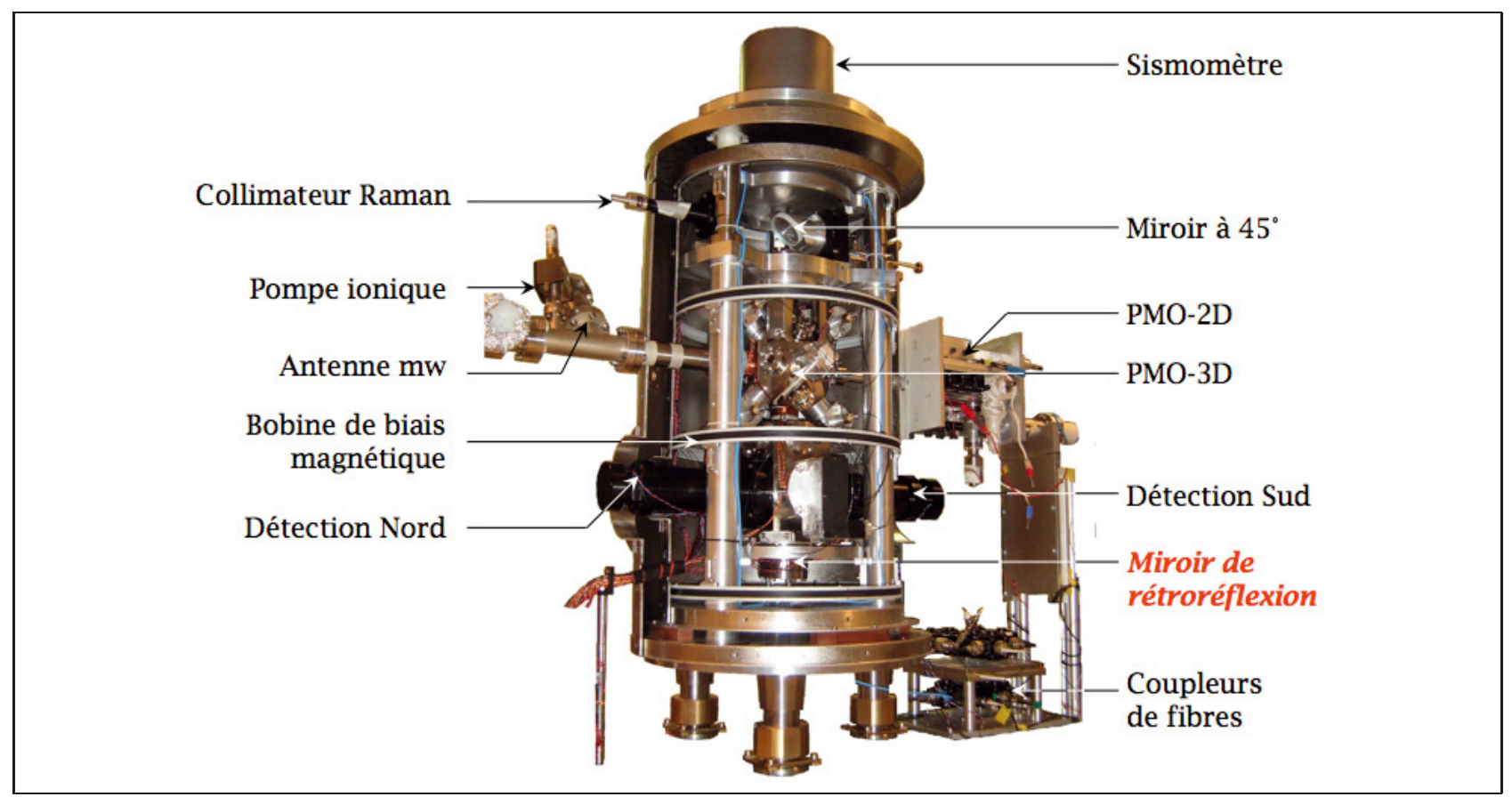

Fig. 12. - Photographie du gravimètre atomique avec deux demi-blindages cylindriques ouverts.

Le principe de mesure repose sur la manipulation cohérente des atomes, réalisée par des transitions Raman stimulées. Cette transition à deux photons où le changement d'état d'impulsion s'accompagne d'un changement d'énergie électronique [43] permet de séparer les paquets d'ondes. Avec un tel outil, on réalise un interféromètre de Mach-Zehnder, comme en optique mais où des atomes remplacent les photons : une première transition sépare les paquets d'ondes, une seconde les redirige l'un vers l'autre, et une dernière recombine les paquets d'ondes des deux chemins et les fait interférer. Les deux états de sortie de l'interféromètre sont identifiés par leur niveau d'énergie électronique, ce qui permet une simple détection par fluorescence [44]. La phase de l'interféromètre, différence de phase entre les deux «chemins », dépend de la différence de phase imprimée sur les atomes par les deux faisceaux laser Raman, séparés en fréquence de l'écart hyperfin. La phase totale s'écrit comme $-k_{\mathrm{eff}} g T^{2}$, où $k_{\text {eff }}$ est le vecteur d'onde effectif défini par les faisceaux lasers qui réalisent les transitions $(\pi / 2-\pi-\pi / 2)$, et $T$ est l'intervalle de temps qui sépare deux impulsions consécutives. La sensibilité croît comme $T^{2}$, ce qui justifie l'intérêt des atomes froids.

\subsection{Dispositif}

Notre gravimètre atomique, usuellement nommé « $\mathrm{CAG}$ », est représenté sur la figure 12 avec la moitié de ses blindages ouverts, ce qui permet de distinguer les différentes parties de l'enceinte à vide et fonctionnalités de l'instrument.

Le CAG réalise une mesure de l'accélération de la pesanteur $g$ avec un nuage d'atomes froids de ${ }^{87} \mathrm{Rb}$ utilisé comme masse d'épreuve. À partir d'un piège magnétooptique 2D (PMO-2D), $10^{8}$ atomes sont chargés en $80 \mathrm{~ms}$ dans un PMO-3D. Suite à une phase de mélasse préparant l'échantillon à une température de $1,7 \mu \mathrm{K}$, les atomes chutent à l'extinction des lasers. Une classe de vitesse d'atomes est alors sélectionnée en utilisant une combinaison d'impulsions micro-ondes et de sélection Raman. L'interféromètre est alors réalisé sur une distance de $17 \mathrm{~cm}$, entre la zone PMO-3D et la double détection symétrique (détection Nord et détection Sud sur la fig. 12). Le collimateur Raman est situé au-dessus de l'enceinte et ses deux faisceaux entrent dans l'enceinte après réflexion sur un miroir à $45^{\circ}$. Afin d'utiliser des transitions Raman contra-propageantes, les faisceaux sont rétroréfléchis sur un miroir situé dans l'enceinte à vide afin d'éviter d'en modifier la phase en traversant des hublots. Seule une paire de deux faisceaux contra-propageants est résonnante avec les atomes, elle est sélectionnée par effet Doppler. On peut ainsi réaliser un interféromètre avec l'une ou l'autre paire de faisceaux Raman que l'on nomme $k_{\uparrow}$ et $k_{\downarrow}$. L'enceinte à vide est placée sur une plateforme d'isolation passive pour limiter les vibrations du miroir de rétroréflection. De plus, un sismomètre placé au sommet de l'enceinte permet de postcorriger ces vibrations et d'améliorer la sensibilité de mesure [45,46].

\subsection{Performances}

Nous avons mis en place un protocole de mesure alternant quatre configurations de mesures, les deux premières diffèrent par le sens du vecteur d'onde effectif $\left(k_{\uparrow}\right.$ et $\left.k_{\downarrow}\right)$ et les deux dernières alternent également le sens de $k_{\text {eff }}$ mais la puissance dans les faisceaux Raman est divisée par 2. Un algorithme utilisant ces quatre mesures successives permettent de mesurer $g$ tout en rejetant la majeure partie des biais systématiques à l'exception du biais lié à l'effet Coriolis et celui lié aux aberrations du front d'onde [47]. Cependant, l'alternance des quatre configurations dégrade la sensibilité d'un facteur $\sqrt{ } 10$. 


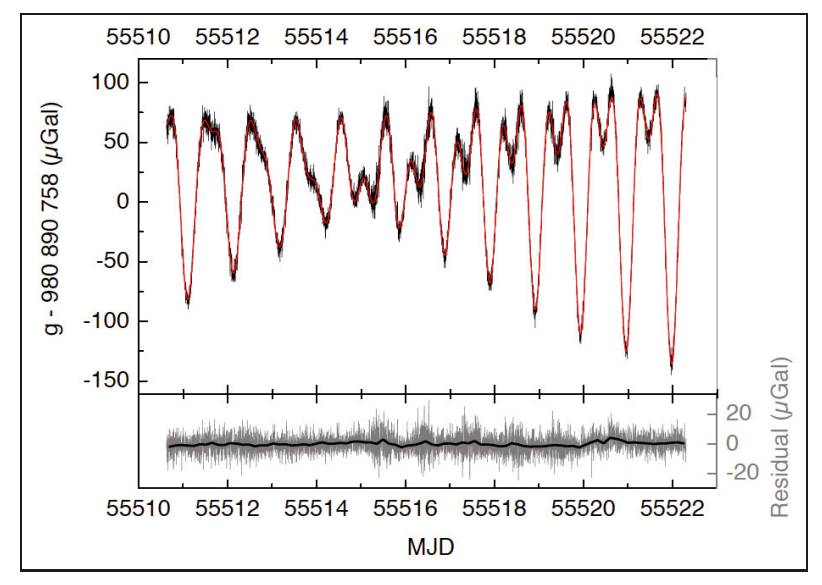

Fig. 13. - Haut : mesure continue des variations de $g$ durant 12 jours exprimés en MJD (Modifian Julian Day); la courbe rouge correspond au calcul du modèle local selon les paramètres du tableau 1. Bas : Le résidu obtenu en soustrayant le modèle est représenté en gris (170 s) et noir (10 000 s).

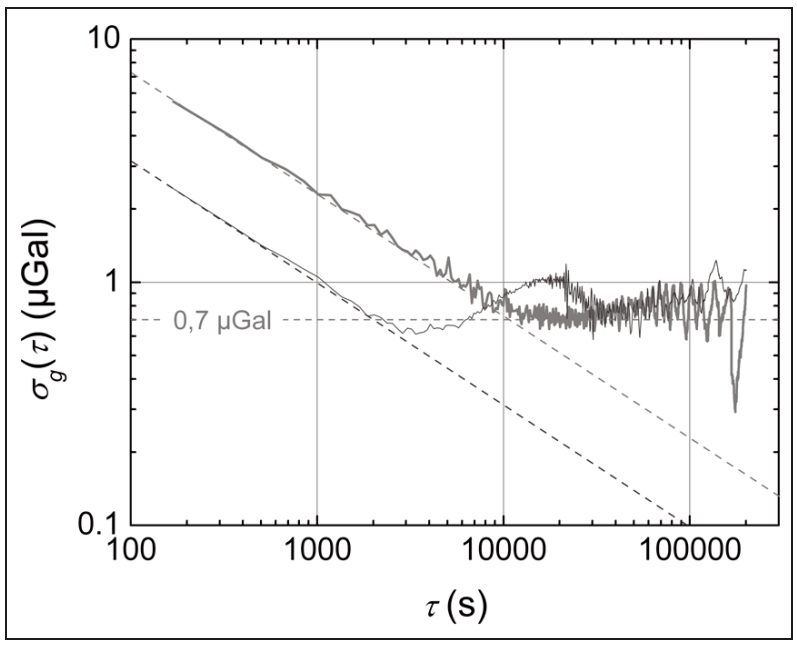

Fig. 14. - Écart type d'Allan du résidu de la mesure de 12 jours (fig. 13). Courbe épaisse grise : pour l'algorithme de mesure complet, trace fine grise foncée : pour un algorithme de mesure ne permettant pas de rejeter le biais dû au déplacement lumineux à deux photons.

La figure 13 montre une mesure continue de $g$ durant 12 jours, en utilisant notre algorithme de mesure qui alterne 100 mesures de chaque configuration, soit $170 \mathrm{~s}$ par point. La mesure est corrigée des variations de la pression atmosphérique et de la position du pôle mais pas des marées. On constate qu'elle correspond fidèlement au modèle local de variations des marées. Le résidu est représenté en bas de figure 13 en gris et en noir pour une moyenne de $10000 \mathrm{~s}$.

L'écart type d'Allan du résidu est représenté en gris sur la figure 14 . Il décroît en $\tau^{-1 / 2}$ et équivaut à une sensibilité de $70 \mu \mathrm{Gal}$ en $1 \mathrm{~s}$ de mesure. La sensibilité est inférieure à $1 \mu \mathrm{Gal}$ après $5000 \mathrm{~s}$ puis atteint à palier à $0,7 \mu \mathrm{Gal}$, comparable au niveau d'exactitude du modèle de marées. Il est ainsi difficile de déterminer si la stabilité long terme de la mesure est limitée par l'instrument ou le modèle utilisé. La seconde courbe en gris foncé
Tableau 2

Bilan des corrections et des incertitudes obtenues pour le gravimètre atomique CAG lors de la comparaison ECAG-2011.

\begin{tabular}{|l|c|c|c|}
\hline Effet & $\begin{array}{c}\text { Biais } \\
(\boldsymbol{\mu G a l})\end{array}$ & $\begin{array}{c}\boldsymbol{u} \\
(\boldsymbol{\mu G a l})\end{array}$ & Référence \\
\hline Alignements & 2,4 & 0,5 & {$[18,41]$} \\
\hline $\begin{array}{l}\text { Référence de } \\
\text { fréquence }\end{array}$ & $-4,6$ & $<0,1$ & {$[18]$} \\
\hline Déphasage RF & 0,0 & $<0,1$ & {$[18,40]$} \\
\hline$v g g$ & $-10,3$ & $<0,1$ & {$[18]$} \\
\hline Auto effet de gravité & $-1,3$ & 0,1 & {$[33]$} \\
\hline $\begin{array}{l}\text { Aberrations du front } \\
\text { d'onde }\end{array}$ & 0,0 & 4,0 & {$[47]$} \\
\hline $\begin{array}{l}\text { Déplacement lumineux } \\
\text { à 1 photon }\end{array}$ & 0,0 & $<0,1$ & {$[40]$} \\
\hline $\begin{array}{l}\text { Déplacement lumineux } \\
\text { à 2 photons }\end{array}$ & $-7,7$ & 0,5 & {$[18,40]$} \\
\hline Zeeman & 0,0 & $<0,1$ & {$[40]$} \\
\hline $\begin{array}{l}\text { Décalage en fréquence } \\
\text { du laser de référence }\end{array}$ & 0,0 & 0,5 & {$[18]$} \\
\hline $\begin{array}{l}\text { Fluctuations des } \\
\text { puissances optiques }\end{array}$ & 0,0 & 1,0 & {$[18]$} \\
\hline $\begin{array}{l}\text { Effet d'indice du } \\
\text { nuage }\end{array}$ & 0,4 & $<0,1$ & {$[18,40]$} \\
\hline Collisions froides & $<0,1$ & $<0,1$ & {$[40]$} \\
\hline TOTAL & $\mathbf{- 2 1 , 1 0}$ & $\mathbf{4 , 3}$ & \\
\hline
\end{tabular}

représente l'écart type d'Allan en utilisant la moitié des données de l'algorithme de mesure. La sensibilité à court terme est meilleure mais ne permet pas d'éliminer le biais dû au déplacement lumineux à deux photons [48] qui fluctue dans l'instrument comme le montre la «bosse » à 20000 s. L'algorithme complet permet de rejeter ce biais et ainsi ses fluctuations.

Les biais non rejetés par le protocole de mesure ont été étudiés pour finaliser le budget d'exactitude de l'instrument et ainsi participer aux comparaisons internationales. Le tableau 2 représente le dernier budget transmis pour la dernière comparaison internationale ayant eu lieu : ECAG-2011 (voir paragraphe 3.5).

Le bilan d'incertitude est dominé par le biais dû aux aberrations du front d'onde. Comme indiqué dans [47], l'expansion balistique des atomes lors de leur chute est la cause principale de ce biais. Nos études actuelles visent à réduire l'incertitude associée en refroidissant plus encore les atomes, pour réduire cette expansion.

\subsection{Mobilité}

Pour comparer les gravimètres, il est nécessaire de déplacer les instruments sur un même site, et surtout d'échanger les positions des gravimètres sur les points de mesure. De plus, le gravimètre ayant été réalisé à l'Observatoire de Paris, et le site de la balance du watt étant situé à Trappes, le gravimètre atomique CAG a été conçu pour être déplaçable.

Le gravimètre CAG est composé de deux parties : l'enceinte à vide, de taille relativement réduite (fig. 12 et 15), et une structure comportant le banc optique 


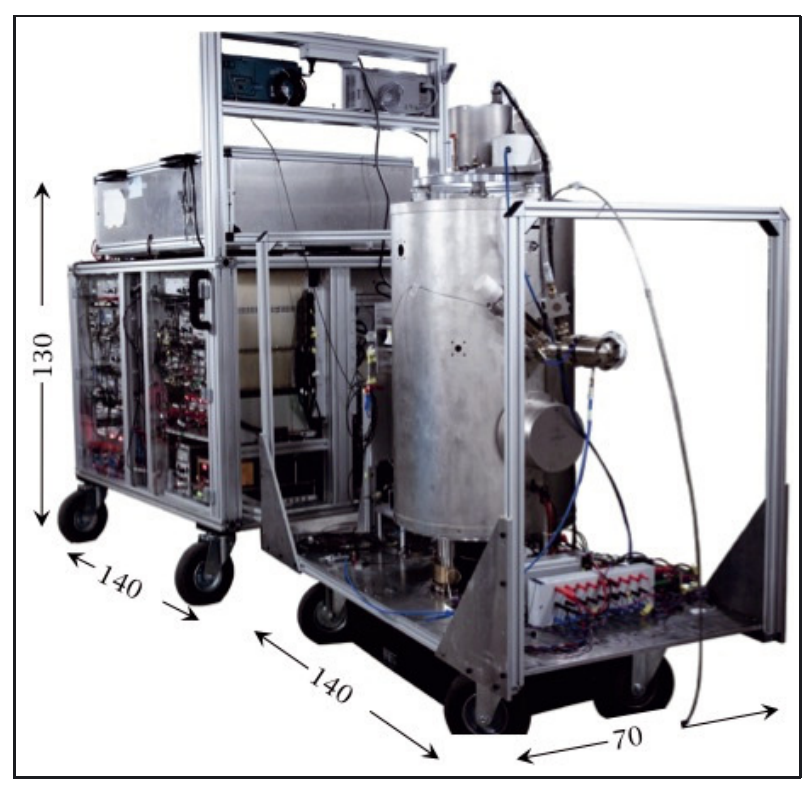

Fig. 15. - Gravimètre CAG en déplacement (les dimensions sont indiquées en centimètres). Au premier plan, l'enceinte à vide. Second plan, l'électronique de contrôle et le banc optique.

et l'électronique de contrôle. Lors de la mesure, ces deux parties sont reliées par des fibres optiques et différents câbles (coaxiaux et d'alimentation électrique). Ces deux parties peuvent être déplacées à l'aide de roues pour mesurer d'autres points sur le site GR ou pour déplacer le gravimètre lors des comparaisons ou encore effectuer des mesures dans d'autres environnements et conditions de mesures.

Nous avons notamment déplacé le gravimètre CAG au LSBB ${ }^{10}$, à Rustrel dans le Vaucluse. L'instrument avait été chargé dans le véhicule la veille du départ puis remis sous tension à l'installation le soir après le trajet. Les pompes de type Getter maintiennent le vide durant ces 30 heures sans alimentation électrique et après une nuit de thermalisation, le gravimètre était opérationnel le lendemain matin pour effectuer des mesures. Notons que cette campagne de mesure nous a permis d'éprouver la sensibilité à court terme de l'instrument en environnement à bas bruit. Sans aucun système d'isolation des vibrations, le gravimètre $\mathrm{CAG}$ a atteint des sensibilités record de $1 \mu \mathrm{Gal}$ sur $100 \mathrm{~s}$ de mesure [49].

\subsection{Comparaisons clefs}

Après l'obtention des premiers signaux interférométriques en 2009, l'instrument a été le premier gravimètre atomique à participer à une comparaison internationale de gravimètres absolus, l'ICAG2009, la dernière organisée au BIPM [50]. Il s'agissait de la première comparaison clef $(\mathrm{KC})$ en gravimétrie. Le résultat final de la comparaison est représenté en figure 16 d'après [50] avec les degrés d'équivalence des gravimètres impliqués.

Les comparaisons ICAG ont jusqu'à présent eu lieu tous les quatre ans, comme les comparaisons européennes

\footnotetext{
${ }^{10}$ LSBB : Laboratoire Souterrain à Bas Bruit.
}

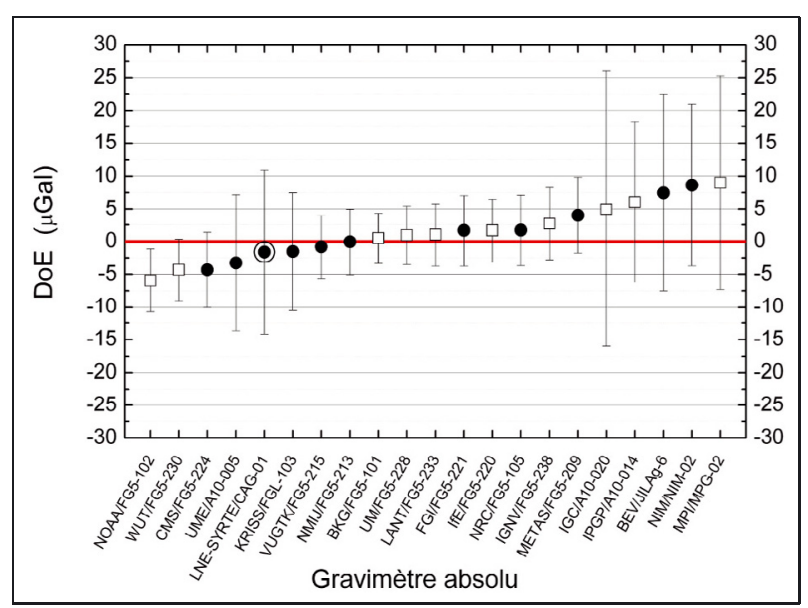

Fig. 16. - Degré d'équivalence(DoE) des gravimètres participants à l'ICAG2009 au BIPM d'après [50]. Les ronds noirs correspondent aux participants à la KC. Les carrés blancs participaient à la comparaison hors KC. Le CAG du LNE-SYRTE est repéré d'un second cercle.

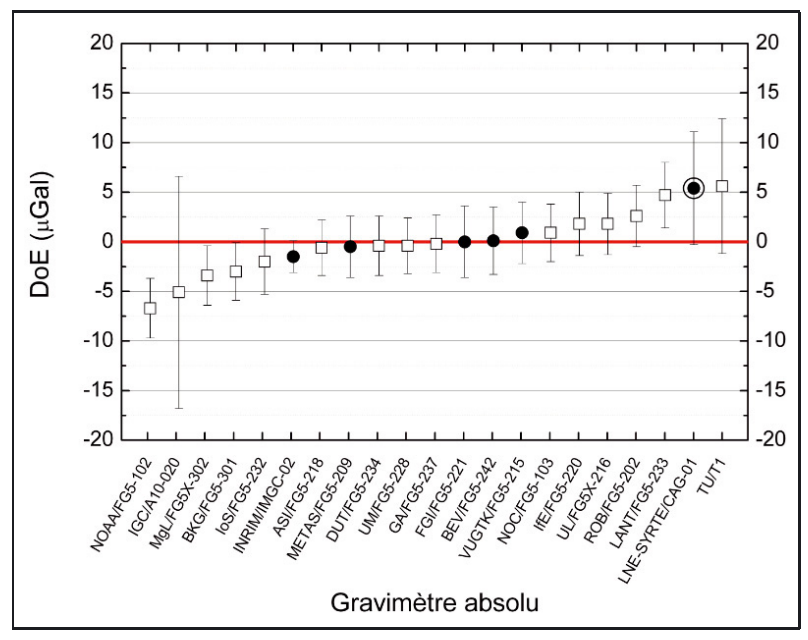

Fig. 17. - Degré d'équivalence (DoE) des gravimètres participants à la comparaison ECAG2011, au Luxembourg d'après [53]. Les ronds noirs correspondent aux participants à la comparaisons clefs (KC). Les carrés blancs participaient à la comparaison hors KC. Le gravimètre CAG du LNE-SYRTE est repéré par un second cercle.

ECAG. L'espacement temporel entre les comparaisons ICAG et ECAG est de deux ans. La figure 17 représente les degrés d'équivalence des gravimètres lors de l'ECAG2011 (également une comparaison clef).

À l'automne 2013, le gravimètre CAG a participé à la comparaison ICAG2013 au Grand Duché de Luxembourg. Les règles d'organisation des comparaisons clefs interdisent de communiquer sur ces mesures avant la validation du rapport «draft $B »$.

\subsection{Améliorations}

Afin de réduire l'incertitude du gravimètre atomique, nos efforts portent actuellement sur la réduction du biais dû aux aberrations du front d'onde. Deux approches sont menées : la première consiste à effectuer une sélection 


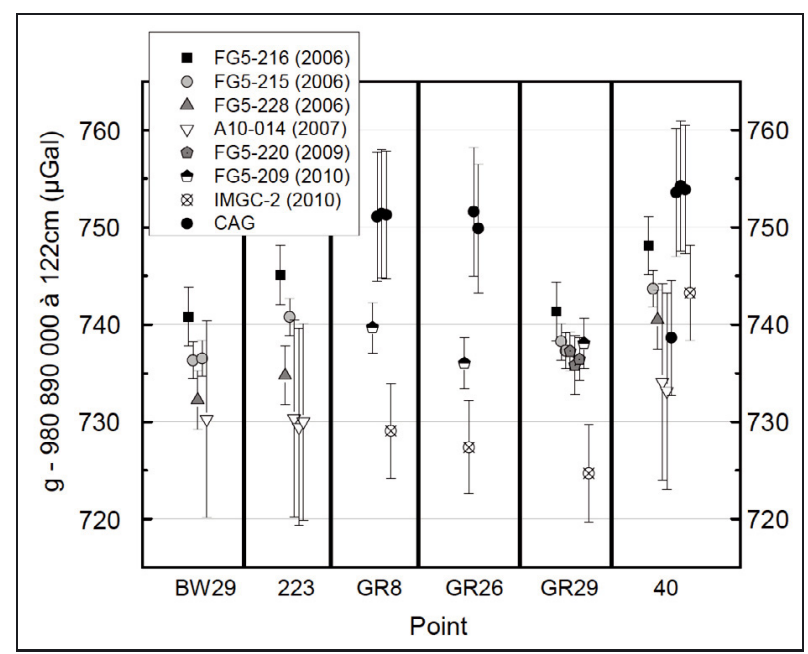

Fig. 18. - Mesures absolues obtenues sur les massifs BW et GR durant les comparaisons organisées sur le site de 2006 à 2010. Les valeurs obtenues avec le gravimètre CAG correspondent aux comparaisons de 2009 et 2010.

Raman horizontale pour étudier la distribution de vitesse horizontale des atomes et réduire l'expansion balistique. La seconde consiste à réaliser un piège dipolaire pour refroidir les atomes jusque quelques dizaines de nanokelvins, afin de réduire l'expansion balistique du nuage atomique.

\section{Résumé des mesures absolues sur le site}

Comme mentionné au paragraphe 2.7, d'autres points du laboratoire ont été mesurés par des gravimètres absolus en plus du gravimètre CAG qui effectue des mesures au point « $40 »$ depuis 2009 . Lors de la réalisation du gravimètre et avant d'assembler la balance du watt, nous avons organisé en 2007 une première comparaison sur les massifs encore relativement inoccupés [36]. Il s'agissait des premières mesures absolues sur le site. Les points centraux ( 2223 » et « $40 »)$ et « $29 »(\mathrm{BW} 29$ et GR29) des deux massifs ont ainsi été mesurés par les gravimètres FG5\#215 du RIGTC ${ }^{11}$, FG5\#216 de 1'Université du Luxembourg et FG5\#228 de Géosciences de l'Université de Montpellier. Six mois plus tard, des mesures ont été faites aux deux centres des massifs par le gravimètre A10\#014 de l'IPGP $^{12}$. Suite à la comparaison ICAG2009, le gravimètre CAG a été installé sur le massif GR au point « 40 » où il a tout d'abord été comparé au gravimètre FG5\#220 du LUH ${ }^{13}$ qui mesurait au point GR29 [51] puis deux autres points, GR8 et GR26 ont été impliqués en 2010 dans la comparaison dans le cadre du projet européen «e-MASS » [52]. Ces mesures sont représentées sur la figure 18 à une hauteur de $122 \mathrm{~cm}$.

Bien que la comparaison avec le gravimètre FG5\#220 $\mathrm{du}$ LUH montrait un accord de $(4,3 \pm 6,4) \mu \mathrm{Gal}$, la

\footnotetext{
11 RIGTC : Research Institute of Geodesy, Topography and Cartography du Geodetic Observatory Pecny de République Tchèque.

12 IPGP : Institut de Physique du Globe de Paris.

${ }^{13}$ LUH : Leibniz Universität of Hannover.
}

comparaison de 2010 a mis en évidence des différences entre le gravimètre $\mathrm{CAG}$ et deux gravimètres à coins de cube (FG5\#209 du Metas et IMGC-02 de l'INRIM) pouvant aller jusqu'à $25 \mu \mathrm{Gal}$. Depuis, nous avons comparé de nouveau l'instrument du laboratoire avec ces deux gravimètres lors de la comparaison ECAG2011, à Walferdange au Luxembourg [53]. Lors de cette comparaison, une seule même station a été visitée par les gravimètres CAG et FG5\#209, la différence entre les mesures en ce point était de 1,2 $\mu \mathrm{Gal}$. De même au point « C5 » la différence obtenue avec les gravimètres FG5\#209 et IMGC-02 était de 0,3 $\mu \mathrm{Gal}$. Ces mesures contredisent les différences obtenues en 2010. La figure 17 montre également que les trois gravimètres comparés en 2010 sont en accord avec le résultat de la comparaison ECAG2011.

Bien qu'il convienne de comparer les mesures obtenues au même moment, les mesures obtenues avec des gravimètres FG5 en GR29 de 2006 à 2010 révèlent une très faible dispersion, tendant à démontrer la stabilité du site.

Le gravimètre CAG effectue des mesures régulièrement au point « 40 ». La dispersion de ses mesures est de $15 \mu \mathrm{Gal}$ comme on le voit sur les mesures obtenues durant les comparaisons de 2009 et 2010.

\section{Conclusion}

Les déterminations de la constante de Planck avec des incertitudes relatives de $10^{-8}$ obtenues avec des expériences de type «balance du watt» nécessitent une détermination de $g$ avec une meilleure incertitude relative. Nous avons utilisé un gravimètre relatif Scintrex CG5 dont les performances nous ont permis de cartographier les laboratoires conçus spécifiquement pour l'expérience de balance du watt au LNE avec une incertitude de $2 \mu \mathrm{Gal}$ et de modéliser les effets de marées avec une incertitude inférieure à $1 \mu \mathrm{Gal}$. Nous avons ainsi pu modéliser les variations de gravité, dans un volume de $50 \mathrm{~m}^{3}$, avec une incertitude inférieure à $3 \mu \mathrm{Gal}$, limitée par les mesures.

La contribution du lien dans l'exactitude est actuellement meilleure que $3 \times 10^{-9}$ dans le volume de $50 \mathrm{~m}^{3}$ et meilleure que $10^{-9}$ entre les centres des deux massifs.

Nous avons conçu un nouveau gravimètre atomique absolu transportable. Les différentes parties qui le composent ont été caractérisées et assemblées au LNE-SYRTE à l'Observatoire de Paris où nous avons obtenu les premiers signaux d'interférence en juin 2009. Depuis, nous avons caractérisé son fonctionnement et étudié les effets systématiques biaisant la mesure de l'accélération de la pesanteur. L'instrument a ainsi été le premier gravimètre atomique à participer la comparaison internationale ICAG. Nous avons depuis participé aux deux autres comparaisons clefs au Luxembourg. Ces comparaisons ont validé le bilan d'incertitude de l'instrument, actuellement compris entre $4 \mu \mathrm{Gal}$ et $5 \mu \mathrm{Gal}$.

À la suite de la comparaison de 2009, le gravimètre atomique a été installé sur le massif dédié à la gravimétrie, à côté de la balance du watt du LNE. Associé 
à la cartographie gravimétrique du site, il permet d'ores et déjà de fournir une valeur absolue de $g$ en continue pour une détermination de la constante de Planck avec une exactitude inférieure à $5 \times 10^{-9}$.

Notre objectif est d'améliorer le gravimètre absolu afin d'atteindre une exactitude de $1 \mu \mathrm{Gal}$. Afin de réduire l'expansion balistique du nuage atomique, un piège dipolaire est en cours de réalisation, dans lequel nous prévoyons de refroidir le nuage par refroidissement évaporatif. L'utilisation d'atomes encore plus froids nous permettra de réduire l'incertitude associée au biais des aberrations du front d'onde. Par ailleurs, la finalisation de l'étalonnage du gravimètre supraconducteur permettra de disposer d'un gravimètre d'une sensibilité comparable au CAG, qui permettra d'étudier la stabilité long terme des mesures du gravimètre atomique. En particulier, un modèle de marées plus fin que le modèle actuellement utilisé sera alors déterminé. Ces différentes améliorations seront éprouvées lors de prochaines comparaisons.

\section{Remerciements}

Les auteurs remercient les services communs du SYRTE qui ont grandement contribué au développement du gravimètre atomique. L'enceinte à vide a été conçue et réalisée par l'équipe mécanique de David Holleville, avec Armel Legrand et Bertrand Venon pour la conception, Jean-Pierre Aoustin, Jean-Jacques Bousquet et Laurent Pelay pour 1'usinage ainsi qu'Annie Gérard et Florence Cornu pour l'assemblage et la mise à vide. Les dispositifs électroniques ont été réalisés par le service électronique de Michel Lours avec Laurent Volodimer et José Pinto Fernandes. Nous remercions également l'équipe informatique de Pascal Blondé avec Gilles Sakoun pour leur aide précieuse. Merci également à David Horville du GEPI pour la conception et la réalisation de la détection du CAG.

Nous tenons également à remercier les collègues participants ou ayant participés au projet «Balance du watt»: Franck Bielsa, Patrick Espel, Matthieu Thomas, Yves Briand, Patrick Juncar, Patrick Pinot, Pierre Gournay, Christian Hauck, Jean David, François Villar, Darine Haddad...

Nous remercions enfin nos collègues Vojtech Palinkas, Jakob Kostelecky, Nicolas Le Moigne, Thomas Jacob, Sébastien Deroussi, Ludger Timmen, Olga Gitlein, Claudio Origlia et Henri Baumann pour leur participation aux comparaisons absolus sur le site du LNE.

Ces travaux ont été réalisés avec le soutien financier du LNE, du CNES, de l'Institut Francilien de Recherche sur les Atomes Froids (IFRAF), de la DGA et de l'Union Européenne (projet FINAQS). Une partie des travaux a été réalisée dans le cadre des JRP e-Mass et kNOW de l'EMRP, avec le soutien financier de l'Union Européenne. L'EMRP est cofinancée par les pays participants à l'EMRP dans le cadre d'Euramet et par l'Union Européenne.

\section{Références}

[1] «Le Système international d'unités », BIPM, $8^{\mathrm{e}}$ édition, 2006.

[2] DAVIS R.S., "The SI unit of mass", Metrologia, 40, 2003, 299-300.

[3] BORDÉ Ch.J., «La lettre de l'Académie des sciences », 20, 2007.

[4] «Résolution 5», 20 CGPM, 1995, 1996, 222.

[5] «Résolution 7», 21 ${ }^{\mathrm{e}}$ CGPM, 1999, 2000, 331.
[6] «Résolution $12 », 23^{\mathrm{e}}$ CGPM, 2007.

[7] BeckeR P., BETTIN H., DANZEBRINK H.-U., GLÄSER M., Kuetgens U., Nicolaus A., Schiel D., De Bièvres P., VALKIERS S. et TAYLOR P., "Determination of the Avogadro constant via the silicon route", Metrologia, 40, 2003, 271-287.

[8] BECKER P., "Tracing the definition of the kilogram to the Avogadro constant using a silicon single crystal", Metrologia, 40, 2003, 366-375.

[9] Genevès G., Gournay P., Gosset A., Lecollinet M., Villar F., Pinot P., Juncar P., Clairon A., Landragin A., Holleville D., Pereira Dos Santos F., David J., Besbes M., Alves F., Chassagne L. et TOPSU S., "The BNM Watt Balance Project", IEEE Trans. Instrum. Meas., 54, 2005, 850-853.

[10] KIBBLE B.P., "A measurement of the gyromagnetic ratio of the proton by the strong field method", In : J.H. Sanders and A.H. Waptra (Eds.) Atomic Masses and Fondamental Constants, 5, Plenum, New York, 1976, 545-551.

[11] Debeglia N., Donsimoni M., Dupont F. et VernouX J.-F., « Expertise géologique, hydrogéologique et gravimétrique d'un site situé au Laboratoire National d'Essais à Trappes (Yvelines) », BRGM/RC-51240-FR, 2001.

[12] Peterson J., "Observations and modeling of seismic background noise", USGS Open file Rept, 1993.

[13] http://www.scintrexltd.com/

[14] Jiang Z., Becker M., Francis O., Germak A., Palinkas V., Jousset P., Kostelecky J., DUPONT F., LeE C.W., Tsai C.L., Falk R., Wilmes H., KopaeV A., Ruess D., Ullrich M.C., Meurers B., Mrlina J., Deroussi S., Métivier L., PAJot G., Pereira Dos SANTOS F., VAN RUYMbeKe M., NASLIN S. et FerRy M., "Relative Gravity Measurement Campaign during the 7th International Comparison of Absolute Gravimeters (2005)", Metrologia, 46, 2009, 214-226.

[15] Jiang Z., Palinkas V., Francis O., Jousset P., Makinen J., Merlet S., BeCKer M., COUlomb A., KESSLER-SchUlZ K.U., SCHUlZ H.R., ROTHLEITNER Ch., Tisserand L. et LeQuin D., "Relative Gravity Measurement Campaign during the 8th International Comparison of Absolute Gravimeters", Metrologia, 49, 2012, 95-107.

[16] Jousset P., VAN RuYMbeke M., Bonvalot S. et DIAMENT M., "Performance of two Scintrex CG3M instruments at the fourth International Comparison of Absolute Gravimeters", Metrologia, 32, 1995, 231-244.

[17] LONGMAN I.M., "Formulas for computing the tidal accelerations due to the moon and the sun", J. Geophys. Res., 64, 1959, 2351-2355.

[18] MERLET S., «Détermination absolue de g dans le cadre de l'expérience de la balance du watt», Thèse de doctorat de l'Observatoire de paris, 2010.

[19] Melchior P., "The tides of the planet Earth", Pergamon press, 1978.

[20] WenZEL H.G., "Tide-generating potential for the Earth", Springer, 1997, 9-26. 
[21] Francis O., «Modèle global des marées océaniques obtenu par inversion de mesures marégraphiques, gravimétriques et altimétriques », Thèse de doctorat, Université Louvain la Neuve, Belgique, 1993.

[22] TAMURA Y., "A harmonic development of the tide generating potential", Bull. d'Inf. Marées Terrestres, 99, 1987, 6813-6855.

[23] Merlet S., Kopaev A., Diament M., Genevès G., LANDRAgin A. et Pereira Dos SANTos F., "Microgravity investigations for the LNE watt balance project", Metrologia, 45, 2008, 265-274.

[24] MerRiam J.B., "Atmospheric pressure and gravity", Geophys. J. Int., 109, 1992, 488-500.

[25] "International Absolute Gravity Basestation Network (IAGBN), Absolute Gravity Observations", Data Processing Standards and Station Documentation (Int. Grav. Com. - WGII : World Gravity Standards), Bur. Grav. Int. Bull. d'Inf. Toulouse, 63, 1988, 51-57.

[26] ToRGE W., "Gravimetry”, de Gruyter, 1989, ISBN 3-11010702-3

[27] TIMMEN L., "Absolute and Relative Gravimetry", In : G. Xu (ed.), Sciences of Geodesy - I, Springer-Verlag, Berlin, Heidelberg, DOI : 10.1007/978-3-64211741-1_1, 1, 2010 .

[28] WAHR J.M., "Deformation induced by polar motion", $J$. Geophys. Res., 76, 1985, 199-214.

[29] Kroner C., Naujoks M., Weise A. et Jahr T., "Detection of small hydrological variations in gravity by repeated observations with relative gravimeters", J. Geod. 10.1007/s00190-007-0202-9, 2007.

[30] Crommen O., Petermans T., Verbeeck K., Meurers B., VAN DaM T., VAN CAMP M., VANCLOOSTER M. et DASsargues A., "Hydrological investigations at the Membach station, Belgium, and application to correct long periodic gravity variations", J. Geophys. Res., 111, 2006, B10403.

[31] http://www.gwrinstruments.com/

[32] Li X. et ChouteaU M., "Three dimensional gravity modeling in all space", Surv. Geophys., 19, 1998, 339-368.

[33] D'Agostino G., Merlet S., LANDragin A. et Pereira Dos SANTOS F., Perturbations of the local gravity field due to mass distribution on precise measuring instruments: a numerical method applied to a cold atom gravimeter, Metrologia, 48, 2011, 299-305.

[34] www.microglacoste.com/fg5.html

[35] Niebauer T.M., Sasagawa G.S., Faller J.E., Hilt R. et KLOPPING F., "A new generation of absolute gravimeter", Metrologia, 32, 1995, 159-180.

[36] Merlet S., Francis O., Palinkas V., Kostelecky J., LE MOIGNE N., JACOBS T. et GENEVÈs G., "Absolute gravimetry measurements at LNE", Terrestrial Gravimetry Static and Mobile Measurements (TG-SMM 2007) Symp., St Petersburg, Russie, 2007, 173-174.

[37] Eichenberger A., Genevìs G. et Gournay P., "Determination of the Planck constant by means of a watt balance”, Eur. Phys. J. Special Topics, 172, 2009, 363-383.
[38] STock M., "Watt balance experiments for the determination of the Planck constant and the redéfinition of the kilogram", Metrologia, 50, 2013, R1-R16.

[39] Cheinet P., «Conception et réalisation d'un gravimètre à atomes froids », Thèse de Doctorat, Université Paris VI, Observatoire de Paris, 2006.

[40] LE GouËT J., «Étude des performances d'un gravimètre atomique absolu : sensibilité limite et exactitude préliminaire », Thèse de doctorat de sciences, Université Paris XI, Observatoire de Paris, 2008.

[41] Pereira Dos Santos F., Le GouËT J., MehlstaÜbler T.E., Merlet S., Holleville D., Clairon A., LANDRAGIN A., "Gravimètre à atomes froids », Revue française de métrologie, 13, 2008, 33-40.

[42] http://syrte.obspm.fr/tfc/capteurs_inertiels/

[43] Bordé Ch.J., "Atomic interferometry with internal state labeling", Phys. Lett. A, 140, 1989, 110-112.

[44] Bordé Ch.J., "Atomic interferometry and laser spectroscopy", Laser spectroscopy X, World Scientic, 1991, 239245.

[45] Le GouËt J., MehlstÄUler T.E., Kim J., Merlet S., Clairon A., Landragin A. et PEREIRA Dos SANTos F., "Limits to the sensitivity of a low noise compact atomic interferometer", Appl. Phys. B, 92, 2008, 133-144.

[46] Merlet S., Le Gouët J., Bodart Q., Clairon A., LANDRAGIN A., PEREIRA Dos SANTOS F. et ROUCHON P., "Operating an atom interferometer beyond its linear range", Metrologia, 46, 2009, 87-94

[47] LOUchet-Chauvet A., FARAH T., BOdART Q., Clairon A., Landragin A., Merlet S. et Pereira Dos SANTOS F., "Influence of transverse motion within an atomic gravimeter", New J. Phys., 13, 2011, 065025.

[48] Gauguet A., Mehlstäubler T.E., LÉvèQue T., Le Gouët J., Chaibi W., Canuel B., Clairon A., Pereira Dos SANTOS F. et LANDRAGIN A., "Off resonant Raman transition impact in an atom interferometer", Phys. Rev. A, 78, 2008.

[49] Farah T., Guerlin C., Landragin A., Bouyer Ph., Gaffet S., Pereira Dos Santos F. et Merlet S., "Underground Operation at Best Sensitivity of the Mobile LNE-SYRTE Cold Atom Gravimeter", Gyroscopy and Navigation, 5, 2014, 266-274.

[50] Jiang Z., Palinkas V., Arias F.E., Liard J., MERLET S., WiLMES H., VitUSHKIN L., ROBERTSON L., Tisserand L., PEReira Dos SANTos F., Bodart Q., FalK R., Baumann H., Mizushima S., MäKinen J., BilKeR-Koivula M., LeE C., CHOI I.M, KARABOce B., Ji W., Wu Q., Ruess D., Ullrich C., Kostelecky J., Schmerge D., EcKl M., Timmen L., Le Moigne N., Bayer R., Olszak T., Agren J., Del Negro C., Greco F., Diament M., Deroussi S., Bonvalot S., Krynski J., SeKowski M., Hu H., Wang L.J., SVITlov S., GermaK A., Francis O., Becker M., INGLIS D. et RoBINSON I., "The 8th International Comparison of Absolute Gravimeters 2009 : the first Key Comparison (CCM.G-K1) in the field of absolute gravimetry”, Metrologia, 49, 2012, 666-684. 
[51] Merlet S., Bodart Q., Malossi N., Landragin A., Pereira Dos SANTOS F., Gitlein O. et Timmen L., "Comparison between two mobile absolute gravimeters : optical versus atomic interferometers", Metrologia, 47, 2010, L9-L11.

[52] Louchet-Chauvet A., Merlet S., Bodart Q., Landragin A., Pereira Dos Santos F., Baumann H., D'Agostino G. et Origlia C., "Comparison of 3 absolute gravimeters based on different methods for the eMASS project", IEEE Trans. Instrum. Meas., 60, 2011, 2527-2532.

Article reçu le 28 décembre 2013, version révisée reçue le 16 octobre 2014.
[53] Francis O., Baumann H., Volarik T., Rothleitner C., Klein G., SeIl M., Dando N., Tracey R., UllRich Ch., Castelein S., Hua H., Kang W., Chongyang S., Songbo X., Hongbo T., Zhengyuan L., PÁlinKás V., KOSTELECKY J., MÄKINEN J., NÄRÄNEN J., MERLET S., Farah T., Guerlin Ch., Pereira Dos Santos F., LE Moigne N., Champollion C., Deville S., Timmen L., Falk R., Wilmes H., Iacovone D., Baccaro F., Germak A., Biolcati E., KRYNSKi J., SeKowski M., Olszak T., Pachuta A., Agren J., Engfeldt A., REUDINK R., INACIO P., MCLAUGHLIN D., SHANNON G., ECKL M., WiLKINS T., VAN Westrum D. et Billson R., "The European Comparison of Absolute Gravimeters 2011 (ECAG-2011) in Walferdange, Luxembourg : results and recommendations", Metrologia, 50, 2013, 257-268. 